\title{
Auroral spectral estimation with wide-band color mosaic CCDs
}

\author{
B. J. Jackel ${ }^{1}$, C. Unick ${ }^{1}$, M. T. Syrjäsuo ${ }^{2}$, N. Partamies ${ }^{3}$, J. A. Wild ${ }^{4}$, E. E. Woodfield ${ }^{5}$, I. McWhirter ${ }^{6}$, E. Kendall ${ }^{7}$, \\ and E. Spanswick ${ }^{1}$ \\ ${ }^{1}$ Physics and Astronomy Department, University of Calgary, Calgary, Canada \\ ${ }^{2}$ School of Electrical Engineering, Aalto University, Espoo, Finland \\ ${ }^{3}$ Finnish Meteorological Institute, Helsinki, Finland \\ ${ }^{4}$ Department of Physics, Lancaster University, Lancaster, UK \\ ${ }^{5}$ British Antarctic Survey, Cambridge, UK \\ ${ }^{6}$ Department of Physics and Astronomy, University College London, London, UK \\ ${ }^{7}$ SRI International, Menlo Park, California, USA
}

Correspondence to: B. J. Jackel (bjackel@ucalgary.ca)

Received: 3 November 2013 - Published in Geosci. Instrum. Method. Data Syst. Discuss.: 23 December 2013

Revised: 28 April 2014 - Accepted: 1 May 2014 - Published: 11 June 2014

\begin{abstract}
Optical aurora can be structured over a wide range of spatial and temporal scales with spectral features that depend on the energy of precipitating particles. Scientific studies typically combine data from multiple instruments that are individually optimized for spatial, spectral, or temporal resolution. One recent addition combines all-sky optics with color mosaic CCD (charge-coupled device) detectors that use a matrix of different wide-band micro-filters to produce an image with several (often three) color channels. These devices provide sequences of two dimensional multispectral luminosity with simultaneous exposure of all color channels allowing interchannel comparison even during periods with rapidly varying aurora. At present color auroral image data are primarily used for qualitative analysis. In this study a quantitative approach based on Backus-Gilbert linear inversion was used to better understand the effective spectral resolution of existing and proposed instruments.

Two spectrally calibrated commercial detectors (Sony ICX285AQ and ICX429AKL) with very different color mosaics (RGB (red, green, blue) vs. CYGM (cyan, yellow, green, magenta)) were found to have very similar spectral resolution: three channels with FWHM (full-width halfmaximum) $\approx 100 \mathrm{~nm}$; a NIR (near infrared) blocking filter is important for stabilizing inversion of both three-channel configurations. Operating the ICX429AKL in a noninterlaced mode would improve spectral resolution and provide an additional near infrared channel. Transformations from arbitrary device channels to RGB are easily obtained through inver-
\end{abstract}

sion. Simultaneous imaging of multiple auroral emissions may be achieved using a single-color camera with a triplepass filter. Combinations of multiple cameras with simple filters should provide $\sim 50 \mathrm{~nm}$ resolution across most of the visible spectrum. Performance of other instrument designs could be explored and compared using the same quantitative framework.

\section{Introduction}

Visible aurora occurs when energetic charges from outer space collisionally excite atmospheric atoms and molecules. These charges move along magnetic field lines connecting the upper atmosphere to more distant regions of the magnetosphere or solar wind. Consequently, observing the spatial distribution and temporal variation of aurora can provide important information about geospace topology and dynamics.

Additional details about source population energy distributions may be obtained if optical observations are spectrally resolved. Emitted photon wavelengths can have a complicated dependence on atmospheric composition and density, but certain combinations of emissions may be used to make useful inferences about the precipitation energy (Rees and Luckey, 1974; Strickland et al., 1989). A list of the most commonly studied auroral emissions is included in Table 1, and will be referred to throughout this study. 
Table 1. Commonly studied visible auroral wavelengths.

\begin{tabular}{cc}
\hline$\lambda[\mathrm{nm}]$ & Source \\
\hline 391.4 & $\mathrm{~N}_{2}^{+} 1 \mathrm{NG}(0,0)$ \\
427.8 & $\mathrm{~N}_{2}^{+} 1 \mathrm{NG}$ \\
470.9 & $\mathrm{~N}_{2}^{+} 1 \mathrm{NG}$ \\
486.1 & $\mathrm{H}_{\beta}$ \\
557.7 & $\mathrm{O}\left({ }^{1} \mathrm{D}-{ }^{3} \mathrm{P}_{2}\right)$ \\
630.0 & $\mathrm{O}\left({ }^{1} \mathrm{D} \rightarrow{ }^{1} \mathrm{~S}\right)$ \\
656.3 & $\mathrm{H}_{\alpha}$ \\
777.4 & OI \\
844.6 & OI \\
\hline
\end{tabular}

Visible emissions are usually dominated by some combination of the 557.7 (green) and $630.0 \mathrm{~nm}$ (red) lines, with most of the remainder coming from $\mathrm{N}_{2}^{+} 1 \mathrm{NG}$ (blue) lines. The total of all near infrared (NIR) emissions may be even larger, but most auroral imagers use detectors with minimal sensitivity outside the range of visible wavelengths. Substantial ultraviolet (UV) emissions are absorbed by the lower atmosphere and can only be observed from space.

An example synthetic auroral spectrum is shown in Fig. 1. This was produced by simulating the effects of a monoenergetic $1 \mathrm{keV}$ electron beam incident on a realistic atmospheric model (Strickland et al., 1999). The results are highly dependent on incident energy, with higher energies penetrating to lower altitudes and exciting different atmospheric constituents than at higher altitudes. Real precipitation distributions are often quasi-Maxwellian, but with characteristic and total energies that can vary considerably as a function of location and time.

Some auroral features, such as premidnight arcs, are essentially constant over intervals on the order of tens of minutes. Other phenomena, such as morning sector pulsating patches, have spatially stable luminosity structures that exhibit quasiperiodic fluctuations on timescales of 1-10 s. During extremely dynamic intervals, such as substorm onset, auroral luminosity can increase by several orders of magnitude in less than a minute. Figure 6 shows an example of multiple rapid variations in broadband auroral intensity during a 1 hour period.

Other significant contributions, such as stars or cloudscattered moonlight, can also exhibit rapid fluctuations. This background spectrum is typically more of a continuum, so that useful estimates can be obtained from measurements adjacent to auroral emission lines. However, errors in background subtraction may be a major concern when attempting spectroscopy with faint or rapidly varying auroral emissions.

The disparate requirements of spatially resolved rapid low-light spectroscopy over a wide field of view cannot be achieved with any single instrument. Many auroral observatories use several different devices with complementary capabilities to acquire multiple data streams, which may be

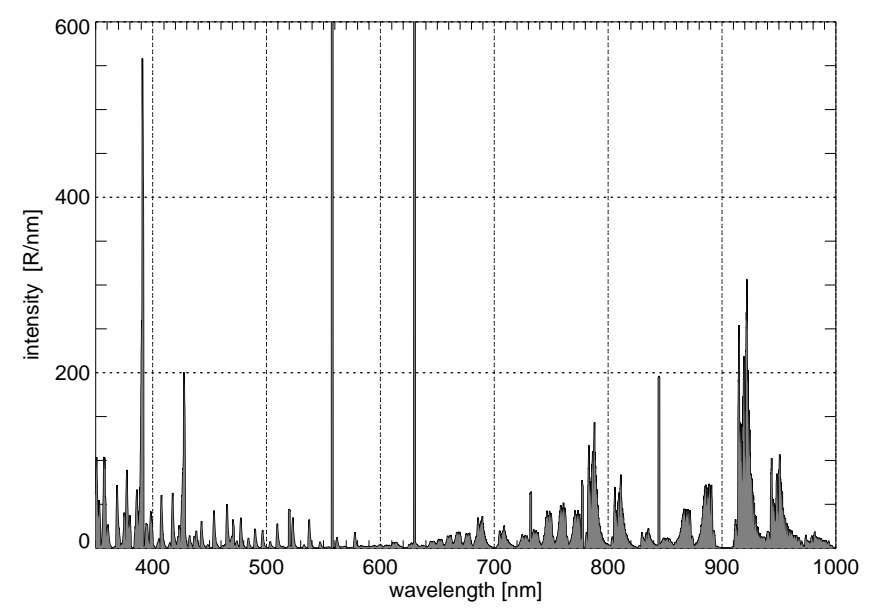

Figure 1. Auroral spectrum during low-activity geomagnetic and solar conditions for a beam of $1 \mathrm{keV}$ monoenergetic electrons with a total energy flux of $1 \mathrm{~mW} \mathrm{~m}^{-2}$. Intensity range is clipped to display spectral structure, $630.0 \mathrm{~nm}$ red-line peak is approximately $810 \mathrm{R} \mathrm{nm}^{-1}$ and $557.7 \mathrm{~nm}$, green line is $1190 \mathrm{R} \mathrm{nm}^{-1}$.

combined to quantify auroral structure as a function of space, time, and wavelength.

One widely used class of auroral instruments is referred to as wide-field or all-sky imagers (ASIs). These devices use fish-eye optics and digital array detectors such as chargecoupled devices (CCDs) to simultaneously observe most or all of the visible sky. CCDs typically have a very broad spectral response, which includes the entire range of visible wavelengths and may extend well into the infrared. The resulting white-light images include contributions from a wide range of photon wavelengths and are useful for rapid observations or detection of faint aurora.

ASIs can be used for quantitative spectroscopy by adding a narrowband (i.e., 1-2 nm) interference filter to isolate specific emission features. This drastically reduces total photon flux reaching the detector, and may require the use of an expensive image intensifier or electron multiplier technology to achieve acceptable levels of signal to noise for the short integration times required to resolve dynamic aurora. A filterwheel (typically with 4-8 filters) can be used to cycle between different passbands in order to observe multiple auroral emissions and appropriate background channels. However, a sequence of long-duration exposures through multiple filters inevitably results in a lack of simultaneity that can be highly problematic when observing aurora that varies rapidly in time. One partial work-around involves the use of two or more identical cameras that can measure different emissions at the same time (Steele and Cogger, 1996; Dahlgren et al., 2008). This approach requires careful calibration to quantify any differences in camera response, but the primary disadvantage is the doubling in cost of an already expensive system. 
Consumer-grade color cameras have become ubiquitous in recent years. Most of these devices use CCDs with a wide-band micro-filter mosaic overlay. Each individual image pixel receives light from a different portion of the optical spectrum (e.g., red, green, or blue); results from neighboring pixels with different filters are interpolated to estimate color (e.g., red, green, and blue) at each pixel location. This process usually involves simultaneous exposure of all pixels, so temporal offsets between color channels are not a problem. Filter rejection will reduce the sensitivity relative to a comparable white-light system, but count rates are still high enough that expensive amplification technology may not be essential. While early versions of this class of detectors were clearly inferior to scientific grade CCDs, recent products provide highquantum efficiency, low noise, and good uniformity.

Color mosaic all-sky imaging systems are increasingly widely used for auroral observations (e.g., Toyomasu et al., 2008). However, most data are typically only used in a qualitative fashion as "quick-look" summaries or for public outreach purposes. It is undoubtedly useful to have visually appealing images that faithfully reflect the appearance of aurora as seen by the human eye. It would be beneficial if wide-band color image data could also be used more quantitatively for scientific applications.

To the best of our knowledge, the auroral science literature on this topic is quite limited. Partamies et al. (2007) present a comparison of wide-band color mosaic imager and narrowband photometer observations, Sigernes et al. (2008, 2009) provide absolute spectral calibration of several different DSLR (digital single-lens reflex) cameras, and Partamies et al. (2012) apply tomography to color mosaic images.

More generally, RGB (red, green, blue) cameras have been used in combination with multiple color filters to estimate daylight and florescent spectra (Nieves et al., 2005, 2007). However, these studies have focused on spectra that are dominated by broad features and it is not clear how their techniques would perform for multiple discrete features such as are typically found in the aurora. More importantly, they do not consider issues regarding nonsimultaneous multichannel measurements of time varying sources.

Given the increasingly common use of all-sky imagers with color CCDs, it is worth exploring the possibility of applying this new source of data to study auroral energetics. Considering existing all-sky imagers, narrowband systems can provide the most information about the characteristic energy of precipitation and white-light observations provide virtually none. The question then becomes, how to quantitatively assess the utility of color ASIs relative to these limiting cases? Our response in this paper is to focus on optimizing spectral resolution. This approach is based on the assumption that a larger number of narrower passbands should lead to more accurate spectral observations and consequently to more accurate energy estimation. An alternate perspective involving direct inversion of precipitation energy is briefly con- sidered in the Discussion, but is otherwise beyond the scope of this paper.

Subsequent sections contain an overview of low-cost color auroral imaging (Sect. 2), detailed spectral calibration of two commonly used color cameras (Sect. 3), a quantitative framework for spectral estimation applied to these two devices (Sect. 4), an examination of some possibilities for future instruments (Sect. 5), and a final discussion (Sect. 6).

\section{Low-cost auroral imaging}

Photographic plates and film were the only options for recording auroral luminosity during more than a century of observations. The introduction of electronic image sensors, such as CCDs, was a revolutionary development that greatly facilitated data acquisition and analysis. However, early CCDs were extremely expensive. Prices for cuttingedge scientific-grade devices remained very high for several decades, and even today a single high-quality CCD may cost thousands of dollars. Equally expensive cooling and electronics are required to achieve maximum performance of a fast readout low-noise camera system. Such an expensive camera usually merits the purchase of the best possible allsky optics, which are inevitably accompanied by custom orders for several large-aperture narrowband interference filters. As a result, the total cost of a complete state-of-the art auroral imaging system can be on the order of USD 50000.

While the performance of high-end CCDs continues to improve, it is important to recognize that low-cost massproduced detectors now perform at a level that would have been called scientific-grade only a few years ago. The proliferation of cameras in consumer electronics means that USD 100 will purchase a high-quantum efficiency low-noise mega-pixel sensor, and prices are falling fast. While very capable, a cheaper CCD only merits similarly inexpensive cooling, electronics, and optics. Consequently, a few thousand dollars is currently sufficient to produce a complete all-sky auroral imaging system.

Significantly lower costs could be reached in return for modest reductions in performance, but at this level ancillary expenses are often more important. Auroral observations usually involve extended operation in polar regions and costly travel to field sites with limited infrastructure. It is arguably not worth allocating the engineering resources to save a thousand dollars on hardware if operating expenses are several times greater.

The first large-scale deployment of low-cost auroral imaging systems was as part of the THEMIS (Time History of Events and Macroscale Interactions during Substorms) ground-based observatory (Donovan et al., 2006; Harris et al., 2008). This project operates white-light all-sky imagers at 20 remote field sites in northern Canada and Alaska. Cameras were purchased from a vendor (Starlight-Xpress) who primarily serves the amateur astronomy community. 
Table 2. Auroral all-sky color imaging system locations. Geographic latitude and longitude are in degrees north and east respectively.

\begin{tabular}{|c|c|c|c|c|c|}
\hline & \multirow{2}{*}{$\frac{\text { Site }}{\text { ID }}$} & \multicolumn{2}{|c|}{ Geographic } & \multirow{2}{*}{$\frac{\text { Device }}{\text { type }}$} & \multirow{2}{*}{$\frac{\text { Operating }}{\text { dates }}$} \\
\hline & & Lat & Long & & \\
\hline \multirow{8}{*}{ 空 } & FSIM & 61.43 & -121.17 & $\mathrm{mx} 7 \mathrm{c}$ & Nov 2006-Apr 2013 \\
\hline & FSMI & 59.98 & -111.84 & $\mathrm{mx} 7 \mathrm{c}$ & Nov 2009-Apr 2013 \\
\hline & GILL & 56.38 & -94.64 & $m \times 7 c$ & Sep 2010-Apr 2013 \\
\hline & PINA & 50.20 & -96.08 & $m \times 7 c$ & Sep 2009-Sep 2010 \\
\hline & RABB & 58.17 & -103.66 & $\mathrm{mx} 7 \mathrm{c}$ & Oct 2006-Feb 2013 \\
\hline & RESU & 74.73 & -94.97 & $m \times 7 c$ & Nov 2007-Nov 2012 \\
\hline & SASK & 52.12 & -107.07 & $\mathrm{mx} 7 \mathrm{c}$ & Sep 2008-Feb 2013 \\
\hline & YKNF & 62.52 & -114.31 & $\mathrm{~h} 9 \mathrm{c}$ & Mar 2009-Apr 2013 \\
\hline \multirow{4}{*}{$\frac{\frac{0}{c}}{\stackrel{\mathscr{J}}{\Sigma}}$} & NYR & 62.34 & -25.51 & $m \times 7 c$ & May 2005-May 2013 \\
\hline & KEV & 69.76 & -27.01 & $\mathrm{mx} 7 \mathrm{c}$ & Dec 2007-May 2013 \\
\hline & MUO & 68.02 & -23.53 & $\mathrm{mx} 7 \mathrm{c}$ & Dec 2007-May 2013 \\
\hline & HEL & 60.17 & -24.93 & $\mathrm{mx} 7 \mathrm{c}$ & Sep 2009-May 2013 \\
\hline \multirow{3}{*}{ 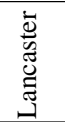 } & HSRN & 62.02 & -6.83 & $m \times 7 c$ & Oct 2009-Aug 2012 \\
\hline & PYKK & 63.77 & -20.54 & $m \times 7 c$ & Oct 2007-Feb 2011 \\
\hline & TJRN & 66.20 & -17.10 & $\mathrm{mx} 7 \mathrm{c}$ & Sep 2009-Mar 2012 \\
\hline SRI & SND & 66.99 & -50.95 & $\mathrm{mx} 7 \mathrm{c}$ & Oct 2008-May 2013 \\
\hline \multirow{3}{*}{$\vec{D}$} & SOD & 67.37 & -26.63 & h9c & Sep 2007-May 2013 \\
\hline & KHO & 78.15 & -16.04 & $\mathrm{~h} 9 \mathrm{c}$ & Sep 2007-May 2013 \\
\hline & KEOPS & 67.88 & -21.07 & $m \times 7 c$ & Jan 2002-Feb 2012 \\
\hline
\end{tabular}

Their camera systems combine thermoelectric cooling and image readout electronics in a convenient package with USB (universal serial bus) interface. Software drivers are provided for Windows and Linux operating systems. THEMIS GBO systems use single-channel Starlight-Xpress MX716 (now rebranded as "Lodestar") cameras with additional all-sky optics. The Sony ICX249AL CCD in these systems has a very broad spectral response spanning the entire visible range of wavelengths, so an additional hot mirror filter was added to reject infrared (see Sects. 3.4, 4.3). For nearly a decade, these systems have gathered $1 \mathrm{~s}$ exposures at a $3 \mathrm{~s}$ cadence whenever the solar zenith angle is greater than $102^{\circ}$. They have proven to be extremely reliable, collecting over 350 million images stored in a lossless (gzipped PGM) format.

Many different Starlight-Xpress systems share the same common software interface, so experience with low-cost white-light cameras for THEMIS could be extended to color mosaic systems. In this study we focus on two different color cameras provided by Starlight-Xpress: the MX7-C (now "Lodestar-C") using the ICX429AKL CCD with a fourchannel color matrix discussed in Sect. 3.2 and the SXV-H9C with a higher-spatial-resolution ICX285AQ three-channel Bayer matrix color CCD discussed in Sect. 3.1. Of course, there are many other available camera models and manufacturers, but these two provide excellent examples of available color mosaics. They have also been used extensively for applications in auroral science at many locations by several different projects. A summary of locations is given in Table 2, with further details provided below to emphasize the organic project evolution facilitated by low-cost devices. Instrument acquisition by groups with different sources of funding may proceed incrementally and deployment plans can be primarily constrained by the availability of existing facilities at remote field sites. The resulting sporadic timelines for development of color auroral imaging given below are in striking contrast to the centralized planning and execution involved in a project like THEMIS.

Researchers at the University of Calgary first developed a prototype system combining THEMIS all-sky optics with an MX7C color camera in 2002. The resulting "Rainbow" systems were field tested in Canada during 2003 and at Svalbard for an optical campaign in early 2004 (Partamies et al., 2007). Rainbow system deployment at eight locations in northern Canada began in 2006 as part of the NORSTAR multispectral imaging (MSI) initiative. Sites at Fort Smith (FSMI), Fort Simpson (FSIM), Gillam (GILL), Pinawa (PINA), and Rabbit Lake (RABB) were operated by the Canadian Space Agency (CSA) Canadian Geospace Monitor (CGSM) program; Resolute Bay (RESU) was funded by the US NSF (National Science Foundation), Saskatoon (SASK) by the University of Saskatchewan, and Yellowknife (YKNF) by the CSA. The Canadian network of Rainbow ASIs operates with $5 \mathrm{~s}$ exposures at a $6 \mathrm{~s}$ cadence in order to maximize sensitivity while facilitating comparison with the $3 \mathrm{~s}$ THEMIS cadence as illustrated in Sect. 3.3. More than 35 million high-resolution "fast"-mode (see Sect. 3.2) images are stored using losslessly compressed PGM files.

Four more Rainbow systems have been deployed as part of the Miracle project (Pulkkinen et al., 1998). The first instrument was installed at Nyrölä (NYR) in 2005, followed by two in 1997 at Kevo (KEV) and Muonio (MUO), then another system at Helsinki (HEL) in 2009. From September 2007 the cameras at KEV and MUO were operated in a mode that provided alternating even and odd frames (see Fig. A2b in Appendix A). After 2009 all four systems operated in "fast" mode with images stored in lossy JPEG format.

Another three Rainbow systems have at been operated by Lancaster University at Pykkvibær in southeastern Iceland (PYKK), at Mánarbakki on the Tjörnes Peninsula in northeastern Iceland (TJRN), and at Húsareyn near Tórshavn in the Faroe Isles (HSRN). The PYKK imager is colocated with the CUTLASS Iceland SuperDARN radar operated by the University of Leicester and shares accommodation and infrastructure with the radar hardware, with the imager mounted in an optical dome installed in the roof of the radar control building. Both the TJRN and HSRN Rainbow imagers are installed in enclosures adapted from industrial modular equipment housings.

Finally, SRI operates a single MX7C system at Sondrestrom (SND) along with the incoherent scatter radar and a wide range of other instruments. Color ASI data provide context on wavelengths not covered by other imagers, and have been found to be particularly effective for distinguishing 
clouds from air glow and aurora. Operating cadence and integration times are the same as for Rainbow.

The H9C camera was first used in Canada for the Dense Array Imaging System (DAISY, see Partamies et al., 2008). This project consisted of three imagers, each with different optics, all providing fields of view smaller than the typical all-sky configuration. The large number of pixels provided by the ICX285AQ was essential for fully resolving small spatial scales during several campaigns. More recently, a single H9C system has been continuously deployed in Yellowknife (YKNF) as part of the CSA AuroraMax project, which provides publicly available real-time high-resolution all-sky color auroral images.

The University College London (UCL) Atmospheric Physics Laboratory also operate their own set of color allsky camera systems for auroral studies; these were developed completely independently of the Rainbow project. Active systems include two H9Cs at Sodankylä (SOD) and Longyearbyen, Svalbard (KHO) and an MX7C at Esrange, Kiruna (KEOPS). These are used primarily as support for the UCL Fabry-Perot interferometers located at all three stations and the scanning Doppler imager (SCANDI) at KHO. These instruments measure winds and temperatures by observing Doppler shifts and emission line profiles of the 630 and $557.7 \mathrm{~nm}$ oxygen emissions. It is essential to have accurate information with regard to cloud cover and auroral structure, so the emphasis has been on qualitative rather than quantitative camera data. For example, contrast stretching is used to enhance auroral features and also the unsharp mask technique is used to emphasize auroral structure and increase the visibility of stars. The cameras are operated continuously during the hours of darkness, controlled by an almanac program. Longer exposures than those used for the Rainbow project (typically $20 \mathrm{~s}$ ) are used with an interval of $2 \mathrm{~min}$ between images. Compared to the images from the previous generation of monochrome all-sky cameras used for this purpose, the improvement in the ability to differentiate between cloud and aurora is dramatic.

\section{Color cameras}

In this study we examine the spectral response of two very different color mosaic CCDs that have been used for auroral imaging. Some basic parameters of these devices are listed in Table 3. Both detectors use an interline architecture in which a single shift moves the contents of each pixel into adjacent masked readout columns. This technique effectively eliminates image smearing during readout and is sometimes referred to as an electronic shutter. Both cameras provide 16 bit pixel data numbers (DN), although effective dynamic range is slightly reduced by a device-dependent constant DC offset that is usually on the order of $1000 \mathrm{DN}$.

Both types of cameras have recently been calibrated in a darkroom facility at the University of Calgary. The primary
Table 3. Characteristics of two color mosaic CCDs.

\begin{tabular}{lll}
\hline Sony model & ICX429AKL & ICX285AQ \\
\hline optical size & $8 \mathrm{~mm}$ diagonal & $11 \mathrm{~mm}$ diagonal \\
chip size $(\mathrm{HxV})[\mathrm{mm}]$ & $7.40 \times 5.96$ & $10.2 \times 8.3$ \\
pixels (effective) & $752 \times 582$ & $1392 \times 1040$ \\
unit cell size $[\mu \mathrm{m}]$ & $8.6 \times 8.3$ & $6.45 \times 6.45$ \\
readout mode & interlaced & progressive \\
color mosaic & CYGM & RGB \\
Starlight-Xpress model & MX7-C & SXV-H9C \\
\hline
\end{tabular}

light source was an incandescent tungsten filament lamp driven by a constant current source. Lamp light was passed through an Acton Research model SP-500i single-grating monochromator with a 1200 line $\mathrm{mm}^{-1}$ grating and $50 \mu$ slit spacings with a $0.65 \mathrm{~nm}$ wavelength resolution. A Newport model 918D-SL-OD1 photodiode with absolute spectral response traceable to NIST (National Institute of Standards and Technology) standards was used to correct for intensity variations in wavelength due to the source blackbody and monochromator grating characteristics.

Two pinholes of approximately $3 \mathrm{~mm}$ diameter separated by about $60 \mathrm{~cm}$ were used to create a collimated beam. This was directly incident on the CCD (with the lens removed from the camera). Image exposures of $400 \mathrm{~ms}$ were acquired for wavelengths ranging from 300 to $1000 \mathrm{~nm}$ at $1 \mathrm{~nm}$ intervals. Some variation in actual exposure time was expected due to the use of a non-real-time operating system; actual jitter was typically less than $1 \mathrm{~ms}$ with rare exceptions on the order of $\pm 10 \mathrm{~ms}(\sim 2 \%)$. Immediately following the CCD measurements, the Newport photodiode was inserted in front of the camera and the wavelength scan was repeated.

Background (dark) levels were estimated from image corners. The observed count statistics were consistent with a shifted Gaussian, which was modeled with a constant bias term added to a Poisson distribution. Results for each device were constant over multiple calibration scans. The ICX285AQ CCD used for this study had a DC bias of roughly 3560 counts and a Poisson mean of 30 counts for a $400 \mathrm{~ms}$ exposure. The ICX429AKL unit had a bias of roughly 1565 counts and a Poisson mean of 25 counts in $400 \mathrm{~ms}$. Our experience with other similar systems is that each device has a unique invariant bias, usually in the range of 1000-4000 data numbers.

Pixels were grouped according to color channel by indexing on a $2 \times 2$ subgrid (see Figs. 2, 4). Any saturated values were excluded from subsequent analysis. Total counts were summed in each channel, background corrected, and normalized using the calibration photodiode measurement.

Very similar calibration configurations were used for all of the results presented here, but there is no guarantee of absolute consistency. This means that, in contrast to Sigernes et al. (2008, 2009), our results cannot be used for absolute sensitivity (i.e., counts/Rayleigh) comparisons between 


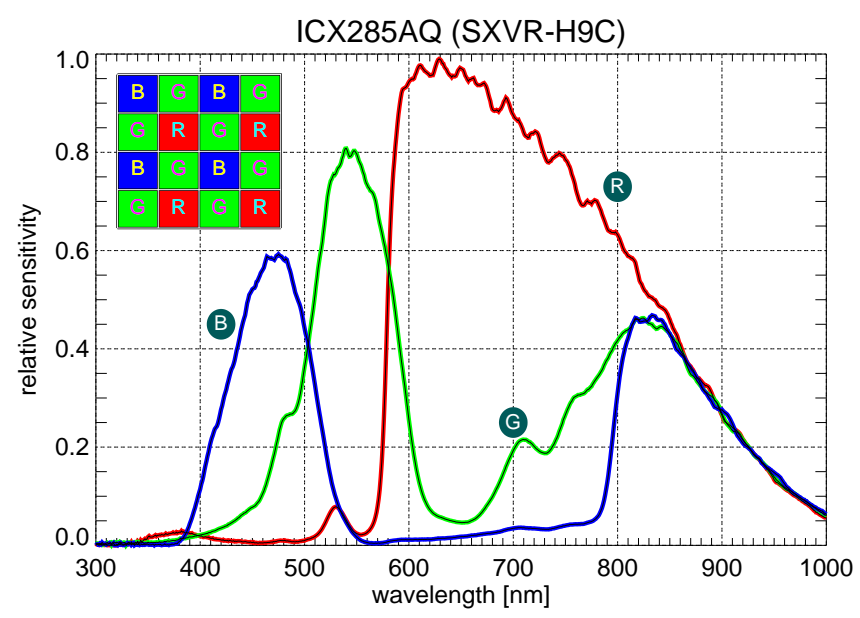

Figure 2. Spectral response for the three-channel (RGB) Sony ICX285HQ CCD with Bayer color mosaic inset.

different cameras. They do, however, provide simultaneous self-consistent results for all color channels on each CCD.

\subsection{Sony ICX285HQ}

The SXV-H9C camera uses a Sony ICX285AQ CCD with three-color RGB microfilters arranged in a standard Bayer matrix pattern (shown in Fig. 2). Three color values at each pixel location can be easily determined by interpolation from neighboring pixels (e.g., Gunturk et al., 2005) with Bayer demosaicing support built into many image processing software packages. The density of green pixels is twice that of red and blue; this configuration can be advantageous for observing aurora, which are often dominated in visible wavelengths by $557.7 \mathrm{~nm}$ atomic oxygen "green-line" emissions. Filter passbands (Fig. 2) are similar to the three different types of color sensitive cones in the human eye. One notable difference is the significant sensitivity in the NIR with essentially identical response in all three channels for wavelengths greater than $800 \mathrm{~nm}$. The standard readout mode uses a progressive scan, so a single frame can contain all active pixels.

\subsection{Sony ICX429AKL}

The MX7C camera has a Sony ICX429AKL CCD with fourcolor cyan, yellow, green, and magenta (CYGM) channels. The microfilter mosaic layout on the CCD is shown in Fig. 3, but typical operating modes produce a slightly different result. Complications arise from the fact that the ICX429AKL readout mode is interlaced (Appendix A), so that all pixels cannot be read out simultaneously. Figure 4a shows the native ("raw") color mosaic along with the output for frames containing only even and odd lines (Fig. 4c, d). Response curves in Fig. 3 were produced by combining results from even and odd frames.

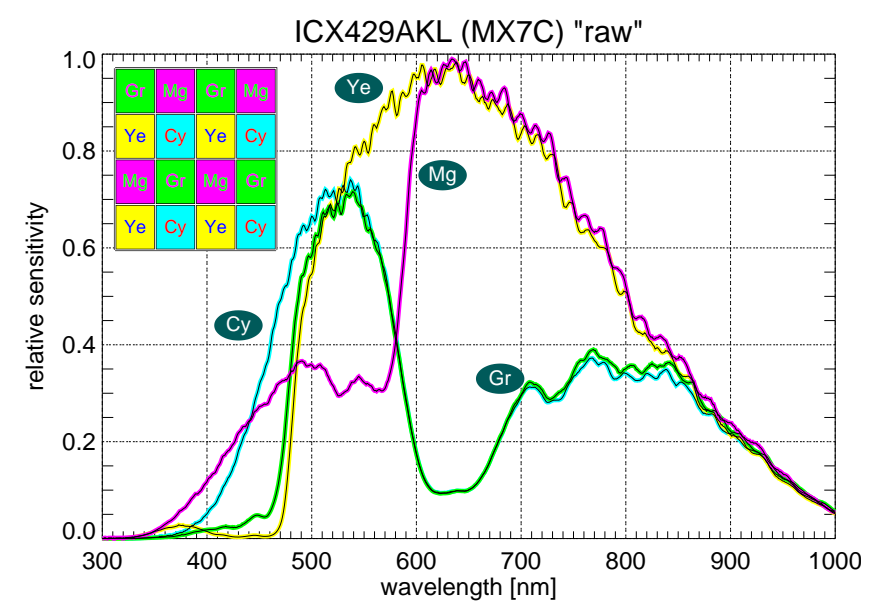

Figure 3. Spectral response of the four-channel (CYGM) Sony ICX429AKL CCD in "raw" mode with color mosaic inset.
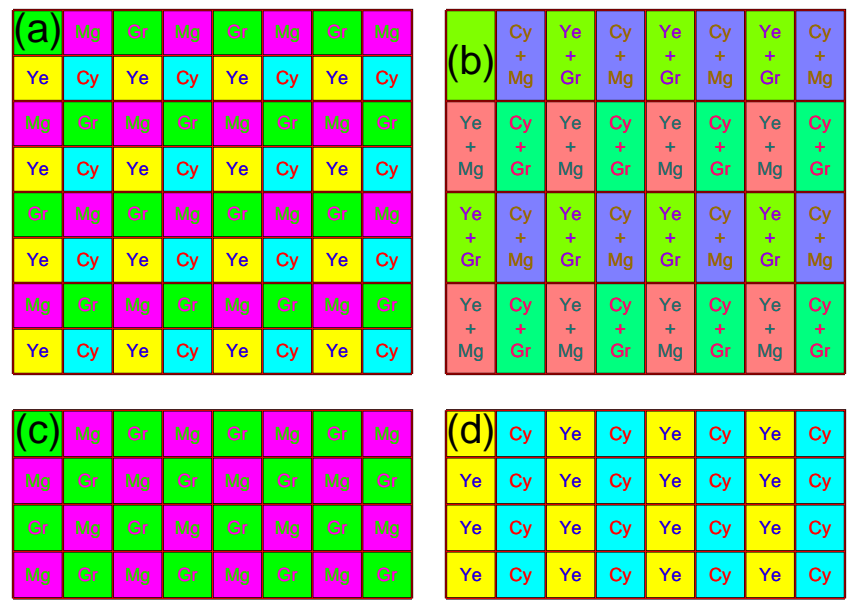

Figure 4. Sony ICX429AKL (a) "raw" full color mosaic, (b) "fast" binned row pairs, (c) even rows, and (d) odd rows.

Separate exposures and readout mean that the even and odd frames will not correspond to exactly the same time interval. Differences on the order of a few seconds may be unimportant for a stable laboratory calibration, but can be a real concern under field conditions when observing highly dynamic aurora. Fortunately, the MX7C camera software does support a "fast" readout mode that sums over adjacent rows (i.e., $1+2,3+4, \ldots$ ) and returns the result as a single half-height frame with an effective color mosaic as shown in Fig. 4b. Data acquired in this "fast" mode were used to produce the response curves shown in Fig. 5.

\subsubsection{Linear independence}

ICX429AKL "fast"-mode data are produced by binning adjacent rows of "raw" pixels. This transformation can be expressed as a matrix multiplication that converts from raw (i.e., cyan $\&$ green) to binned (i.e., cyan + green) color 


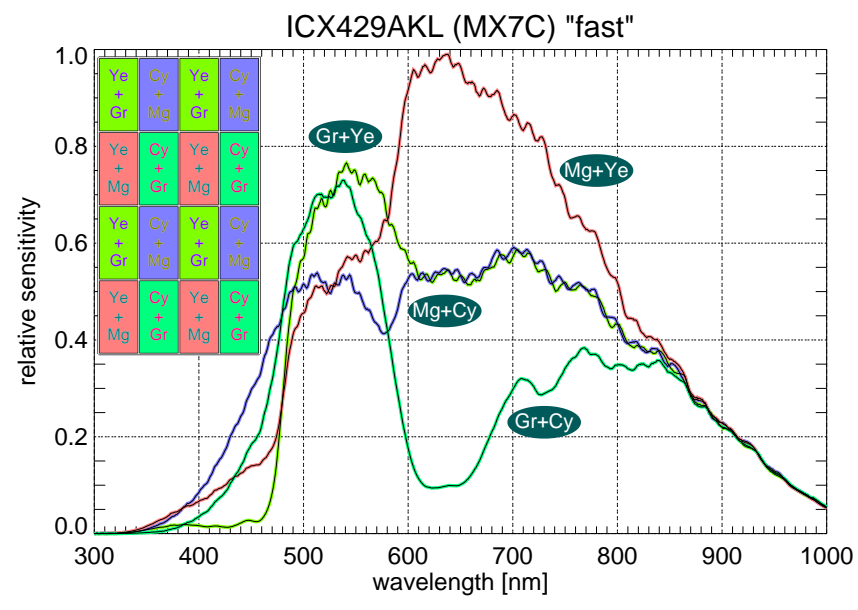

Figure 5. Spectral response of the four-channel (CYGM) Sony ICX429AKL CCD in "fast" mode.

channels:

$$
\left[\begin{array}{llll}
0 & 1 & 1 & 0 \\
1 & 0 & 0 & 1 \\
0 & 1 & 0 & 1 \\
1 & 0 & 1 & 0
\end{array}\right]\left[\begin{array}{c}
\mathrm{Cy} \\
\mathrm{Ye} \\
\mathrm{Gr} \\
\mathrm{Mg}
\end{array}\right]=\left[\begin{array}{c}
\mathrm{Gr}+\mathrm{Ye} \\
\mathrm{Mg}+\mathrm{Cy} \\
\mathrm{Mg}+\mathrm{Ye} \\
\mathrm{Gr}+\mathrm{Cy}
\end{array}\right],
$$

which naturally suggests the possibility of reversing the process in order to obtain the four underlying raw channels from four observed fast channels. Unfortunately, the $4 \times 4$ transformation matrix is singular (zero determinant) and cannot be inverted, meaning that the row binning process is apparently irreversible. This kind of analysis implicitly assumes that the raw color channels are orthogonal, but the validity of this assumption is not obvious given the response functions in Fig. 3.

\subsection{THEMIS/Rainbow sensitivity comparison}

The THEMIS all-sky imager network uses Starlight Xpress model MX7 (now "Lodestar") cameras with Sony ICX429AL CCD detectors. These are monochrome devices that are identical to the ICX429AKL CCD, except without the microfilter mosaic. Consequently, every pixel in the ICX429AL has an identical very broad (panchromatic) spectral response. This is ideal for maximizing the photon count rate under low-light conditions, but data cannot be used to distinguish between different emission wavelengths. Like the MX7C, the MX7 is also an interline system with driver software that can either read alternate rows or the sum of row pairs ("fast" mode). The THEMIS optics project an all-sky image onto a $512 \times 512$ pixel subregion of the CCD that is read in fast mode with $2 \times 2$ binning to produce a $256 \times 256$ array of approximately square superpixels. Images are acquired at a $3 \mathrm{~s}$ cadence with $1 \mathrm{~s}$ exposures; this $33 \%$ duty cycle reflects a combination of constraints in the camera, serial communications (USB 1.1), and a relatively low-power system computer.
The MX7C-based "Rainbow" systems currently operated by the Calgary and Lancaster groups use all-sky optics that are essentially identical to those in the THEMIS system; both systems use CCDs with identical dimensions and pixel density. Rainbow systems operate at a slower $6 \mathrm{~s}$ cadence with longer $5 \mathrm{~s}$ exposures designed to compensate for anticipated losses due to the color mosaic microfilters. Stored image frames consist of $512 \times 256$ rectangular superpixels with four "fast" color channels as described previously. During post-processing these multichannel images can be binned by column pairs to produce $256 \times 256$ superpixels with the same geometry as THEMIS. Each superpixel is composed of four device-level ("raw") pixels with the same channel combination in every case: $\mathrm{Cy}+\mathrm{Gr}+\mathrm{Ye}+\mathrm{Mg}$. The result is a monochrome image with a very wide spectral response that is an average of the four mosaic color passbands.

A monochrome Rainbow image is convenient for certain kinds of analysis that do not require spectral information (e.g., star tracking). It can also be used for a comparison of the relative sensitivity of the two systems. For several years (2009-2013) a Rainbow system has been operating near Fort Smith in northern Canada at a field site that is only a few kilometers from a THEMIS system. Both cameras are looking towards local zenith, but are oriented along slightly different azimuths. A pixel-by-pixel comparison would require precise determination of both camera orientations followed by careful mapping into a common coordinate system.

For this study we shall consider only frame average count rates as a robust measure of whole system response that are not sensitive to orientation errors or minor inhomogeneities in CCD response. Count rates from the two different cameras are usually very highly correlated, as illustrated by an hour of auroral data shown in Fig. 6. The relatively high (87 \%) Rainbow to THEMIS count rate ratio is presumably due to three of four color channels having transmission maxima near the brightest auroral green-line $(557.7 \mathrm{~nm})$ emissions. Although the Rainbow systems do have a lower cadence than THEMIS (6 versus $3 \mathrm{~s}$ ) they have a much higher $(250 \%)$ effective duty cycle (5 versus $1 \mathrm{~s}$ exposures). As currently operated, a Rainbow camera can produce white-light images with more than twice the number of counts from THEMIS over the same interval. This should improve the ability to detect faint structures, albeit at the cost of temporal resolution.

The THEMIS and monochrome Rainbow spectral response functions are similar but not identical. This means that relative changes in different auroral emissions may result in changes to the count rate ratio. In fact, Rainbow to THEMIS count rate ratios (not shown) varied by approximately $10 \%$ over the interval in Fig. 6 with the higher ratios tending to correspond to higher count rates. This is consistent with an increase in characteristic precipitation energy causing a change in the relative intensity of different auroral emissions. A quantitative analysis of changing precipitation is beyond the scope of this paper, and will be the focus of a subsequent study. For now, we simply note that a basic 


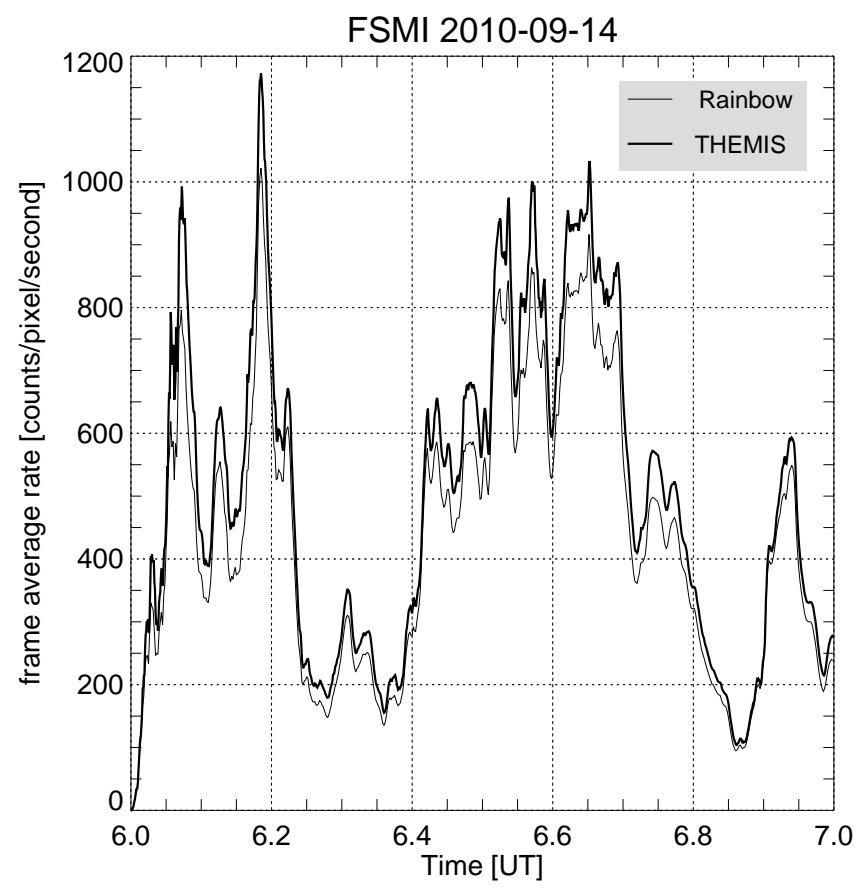

Figure 6. Response of colocated Rainbow and THEMIS imagers at Fort Smith during an active period from 06:00 to 07:00 UT on 14 September 2010. Frame average counts are divided by exposure times of $1 \mathrm{~s}$ for THEMIS and $5 \mathrm{~s}$ for Rainbow to produce comparable rates. THEMIS data (thick line) are plotted at $3 \mathrm{~s}$ intervals and Rainbow (thin line) at $6 \mathrm{~s}$ intervals; timing differences are not visible on this 1-hour interval. Results are very highly correlated $\left(r^{2} \approx 0.994\right)$ with a ratio of $87 \%$.

comparison of two similar white-light channels may provide some information about auroral energetics. If so, it is reasonable to expect that multiple wide-band channels from a color CCD might be even more useful.

\subsection{Infrared rejection}

Dark-room results were obtained using the simplest possible configuration: light from a calibration source directly incident on the thermal glass window in front of each CCD. For field operations there are usually several additional optical elements. Most of these are part of the fish-eye lens system required to image a hemispherical (all-sky) field of view and project it onto the CCD. While there can be significant losses due to reflections between multiple glass surfaces, the overall transmission for these components should be nearly uniform across the visible spectrum.

The THEMIS and Rainbow systems also have an additional filter (Fig. 7) to reject NIR photons above $750 \mathrm{~nm}$. This hot mirror was included to reduce the effects of chromatic aberration over a wide range of wavelengths. Limiting the input light to visible wavelengths was intended to reduce undesirable image blurring. One drawback of this particular filter is the substantial $(50 \%)$ attenuation at shorter wave-

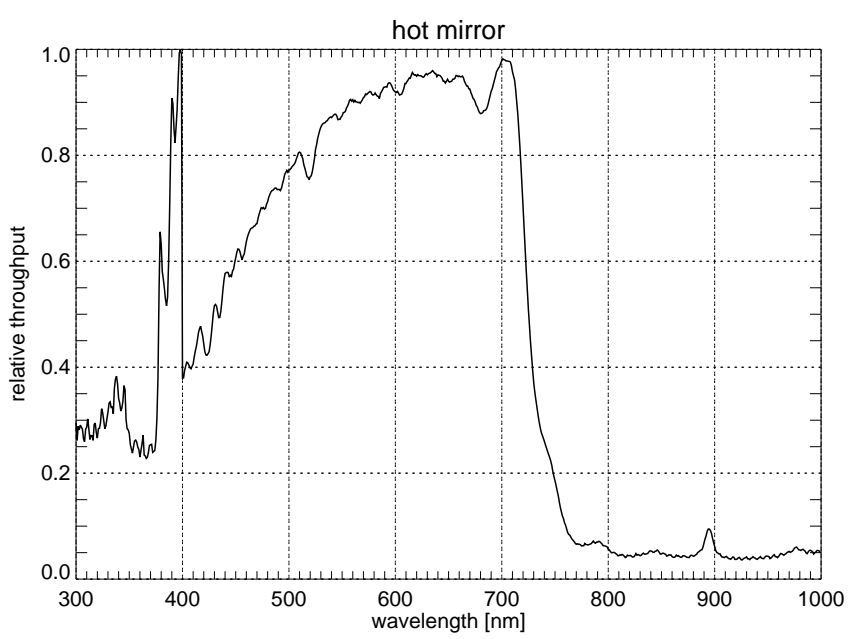

Figure 7. Transmission for infrared blocking filter (hot mirror) used in THEMIS and Rainbow cameras. Feature at $380-400 \mathrm{~nm}$ is a calibration artifact that does not significantly impact analysis results.

lengths, which is unfortunate considering the importance of blue (e.g., 391.4 and $427.8 \mathrm{~nm}$ ) auroral emissions. It could also be argued that NIR auroral features are a sufficiently important source of information so as to outweigh the deficits of slightly less well-focused images. One of the original motivations for this study was a comparison of THEMIS spectral response with and without the hot mirror, and the possibility of differencing data from two different cameras to estimate the NIR contribution.

Given that NIR-blocking filters have been used in many of the systems considered in this study, their effect is included in most of the analysis that follows. This is done using modified CCD response functions produced by multiplying calibrated sensitivity for each device (shown in Figs. 2, 3, and 5) by the hot mirror transmission curve in Fig. 7. We did also repeat the analysis without NIR blocking and found some interesting differences that are explored further in Sect. 4.3.

\section{Spectral estimation}

An ideal color camera would generate images that are perceived by the human eye to be indistinguishable from the source. Practically speaking, this usually means acquiring a rectangular array of $N_{x} \times N_{y}$ pixels, each of which has a RGB triplet of color intensities. A properly displayed image frame should produce the same retinal response as when observing the original scene. The field of color studies is quite complex and requires an understanding of topics including basic optics, biology, and human perception. In this study we deliberately chose to work only with very simple models of color. Our primary focus is the exploration of possibilities for dealing with color CCDs as multispectral detectors. 
To start, consider an ideal linear detector with three different channels. The channel spectral passbands are chosen to exactly match the wavelength sensitivity of the three different types of cones $c_{k}=\left[c_{1}, c_{2}, c_{3}\right] \approx[R, G, B]$ in the human eye. Measurements from this detector $m_{k}=\left[m_{1}, m_{2}, m_{3}\right]$ should be identical to the cone's response. The Bayer matrix CCD in Sect. 3.1 is a good approximation to this ideal detector, with three measurement channels that correspond to the primary colors:

$\left[m_{1}, m_{2}, m_{3}\right] \approx\left[c_{1}, c_{2}, c_{3}\right]$.

Differences between real and ideal detectors can be modeled by some kind of transformation to convert from measurements to true color. In general, there can be more than three measurement channels, and a different functional form may be needed for each color:

$c_{i}=f_{i}\left(m_{1}, m_{2}, \ldots, m_{j}\right)$.

Many transformations can be usefully expressed as linear combinations of measurements $m_{j}$ from $N_{\mathrm{m}}$ measurement channels; multiplying by weighting coefficients $d_{i j}$ to get $N_{\mathrm{c}}$ color channel estimates $c_{i}$ :

$c_{i}=\sum_{j} d_{i j} m_{j}$,

which can be written more compactly as a matrix multiplication,

$\mathbf{c}=\mathbf{D} \mathbf{m}$.

For an ideal three-channel Bayer RGB mosaic the weights D might be a $3 \times 3$ identity matrix, with a CYGM mosaic requiring a more complicated $3 \times 4$ matrix.

In auroral physics, color is really only important as a measure of spectral features corresponding to interesting atomic and molecular emissions. From this perspective, a more useful activity would be to somehow combine multichannel observations in order to estimate spectral intensity $\tilde{s}(\lambda)$ at specific wavelengths of interest; i.e.,

$\tilde{s}(\lambda)=\sum_{n} d_{n}(\lambda) m_{n}$.

We can not reasonably expect to recover high-resolution spectra from a small number of wide-band observations. It is, however, useful to explore the merits of this approach to dealing with color mosaic CCD data.

In general, a spectrum $s(\lambda)$ may be observed using a linear device with $N_{m}$ different measurement channels. Each channel may have a different spectral response given by a "kernel": $K_{i}(\lambda)=K\left(\lambda_{i}, \lambda\right)$, where $\lambda_{i}$ is some characteristic (i.e., peak or average) wavelength corresponding to each channel. The result will be $N_{\mathrm{m}}$ measurements $\tilde{m}\left(\lambda_{i}\right)$, each with some noise contribution $n\left(\lambda_{i}\right)$.

$\tilde{m}\left(\lambda_{i}\right)=\int K\left(\lambda_{i}, \lambda^{\prime}\right) s\left(\lambda^{\prime}\right) d \lambda^{\prime}+n\left(\lambda_{i}\right)$
In an ideal instrument the measurement kernels should be extremely narrow (i.e., Dirac delta functions) and the noise negligible $(n \approx 0)$, so the observations would be an accurate measure of the original source spectrum.

$\tilde{m}\left(\lambda_{i}\right)=\int \delta\left(\lambda_{i}-\lambda^{\prime}\right) s\left(\lambda^{\prime}\right) d \lambda^{\prime}+n\left(\lambda_{i}\right) \approx s\left(\lambda_{i}\right)$

For wide-band color CCDs the kernels are definitely not delta functions and Poisson noise is unavoidable.

Fortunately, this general class of problems arises in many different applications and has been addressed in detail by numerous authors (e.g., Rodgers, 1976, 1990; Menke, 1989; Aster et al., 2005; Tarantola, 2005). Methods for solving these problems are variously referred to as linear inversion, retrieval, or estimation; the shared goal is to infer useful results from a limited number of nonideal observations in the presence of noise.

One effective procedure begins by discretizing the integral in Eq. (7) and replacing it with a finite sum:

$\tilde{m}\left(\lambda_{i}\right) \approx \sum_{j} q_{j} K\left(\lambda_{i}, \lambda_{j}\right) s\left(\lambda_{j}\right) \Delta \lambda+n\left(\lambda_{i}\right)$,

where $q_{j}$ are quadrature weights (i.e., trapezoidal rule, Simpson's rule). This leads to a matrix representation,

$\tilde{\boldsymbol{m}}=\mathbf{K s}+\mathbf{n}$,

which is not solvable in general because $\mathbf{K}$ may not be a square matrix and thus cannot be directly inverted. A solution can, in principle, be obtained by applying the pseudo-inverse

$$
\begin{aligned}
{\left[\mathbf{K}^{T} \mathbf{K}\right]^{-1} \mathbf{K}^{T} \tilde{\boldsymbol{m}} } & =\mathbf{D} \tilde{\boldsymbol{m}} \\
& =\mathbf{D K} \mathbf{s}+\mathbf{D n} \\
& =\mathbf{A s}+\mathbf{e}=\tilde{\boldsymbol{s}},
\end{aligned}
$$

where rows of the matrix D in Eq. (11) are called "contribution functions" (also referred to as inverse response kernels) that indicate what combination of observations should be used to estimate the spectrum at a given wavelength.

Rearranging terms shows that the recovered spectral estimate $\tilde{\boldsymbol{s}}$ consists of two parts (Eq. 12). The first term is a synthetic measurement of the true spectrum as viewed through a set of "averaging" or "windowing" functions $A\left(\lambda_{i}, \lambda\right)$ constructed from a combination of the contribution functions and the measurement kernels. Ideally, the averaging functions $A$ would be extremely narrow and $\mathbf{A}$ would be an identity matrix. In practice each averaging function $A_{i}(\lambda)$ will have some finite width corresponding to the effective resolution limit. A very broad averaging function means that the retrieval process can only recover a highly smoothed version of the underlying spectrum.

The second part of Eq. (12) is an error term arising from a combination of contribution functions and measurement noise. In practice the actual noise values $n_{i}$ are unknown, so 
we must use estimates $\tilde{n}_{i}$ (e.g., $\tilde{n}=\sqrt{m}$ for Poisson noise) to determine the retrieval error,

$\tilde{e}_{i}=\sqrt{\sum_{j} d_{i j}^{2} \tilde{n}_{j}^{2}}$

which should ideally be made as small as possible. Unfortunately, resolution and error are inextricably connected, and it is usually necessary to accept some tradeoff with suboptimal resolution in return for moderate levels of error.

One popular class (Menke, 1989; Aster et al., 2005) of linear retrieval techniques, called Tikhonov regularization, applies smoothness constraints to the recovered profile $\tilde{s}(\lambda)$ in order to damp spurious oscillations driven by measurement noise. An alternative formalism (Backus and Gilbert, 1968, 1970; Parker, 1972) attempts to simultaneously minimize the retrieved variance along with the "spread" $\left(q_{2}\right)$ of the resolution functions,

$q_{2}(\lambda)=12 \int\left(\lambda-\lambda^{\prime}\right)^{2} A\left(\lambda, \lambda^{\prime}\right)^{2} d \lambda^{\prime}$,

to obtain contribution functions given by

$\mathbf{d}(\lambda)=\frac{\mathbf{k}^{T}[\mathbf{Q}(\lambda)+\mu \mathbf{C}]^{-1}}{\mathbf{k}^{T}[\mathbf{Q}(\lambda)+\mu \mathbf{C}]^{-1} \mathbf{k}}$,

where $\mathbf{k}$ is a column vector of the kernel areas $\left(k_{i}=\right.$ $\left.\int K_{i}(\lambda) d \lambda\right), \mathbf{C}$ is the measurement covariance matrix, $\mathbf{Q}(\lambda)$ is the spread matrix

$Q_{i j}(\lambda)=12 \int\left(\lambda-\lambda^{\prime}\right)^{2} K_{i}\left(\lambda^{\prime}\right) K_{j}\left(\lambda^{\prime}\right) d \lambda^{\prime}$,

and $\mu$ is a Lagrange multiplier to select between the extremes of high resolution $(\mu=0)$ and low noise $(\mu=\infty)$.

The Backus-Gilbert approach is more computationally intensive than other regularization techniques, but provides a clear picture of how error and resolution respond to a single control parameter. It is particularly useful for exploring the effective resolution of a hypothetical set of measurements, and how changing measurement details might improve the amount of retrieved information.

For cases with a large set of distinctly many different measurement kernels the $\mu$ parameter can be chosen to make significant changes in the retrieval results. For the case of a three- or four-channel color camera $\mu$ has very little effect, as it is simply not possible to construct very different combinations of kernels. An example showing spread versus noise for a wide range of $\mu$ is given in Fig. 8. The tradeoff curve has the characteristic shape, but both spread and noise vary only over an extremely small range. This is true even for much smaller or larger values of $\mu$. Consequently, in this study we restrict our analysis to a nominal value of $\mu=1$.

\subsection{ICX285AQ (H9C)}

Backus-Gilbert analysis was carried out using the three ICX285AQ response curves from Fig. 2 as measurement kernels. Resolution and contribution functions were calculated

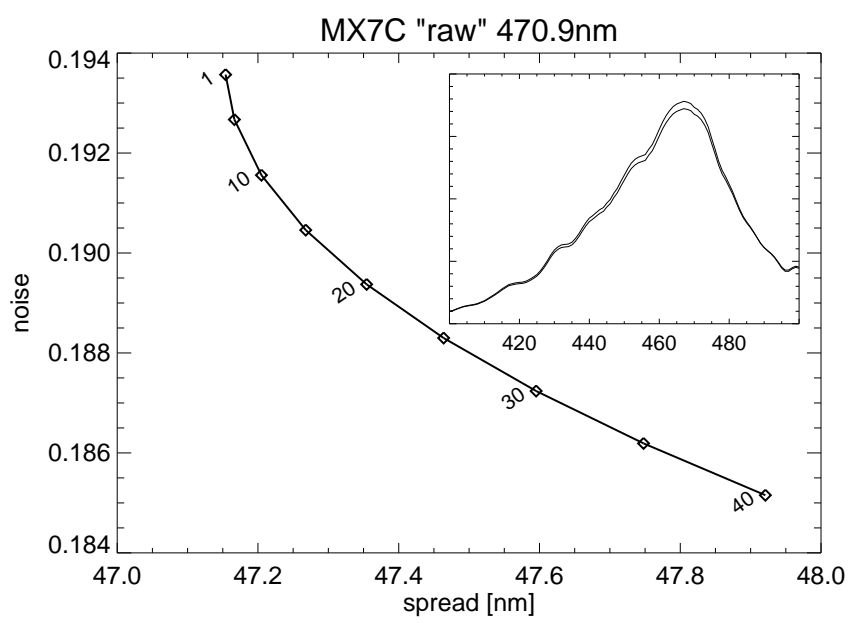

Figure 8. Backus-Gilbert tradeoff curve with inverse relationship between resolution function spread and retrieval error. Results were obtained using MX7C "raw" kernels with a retrieval wavelength of $470.9 \mathrm{~nm}$ and $\mu$ ranging from 1 to 40 . Inset shows nearly indistinguishable resolution functions for $\mu=1$ and $\mu=40$.

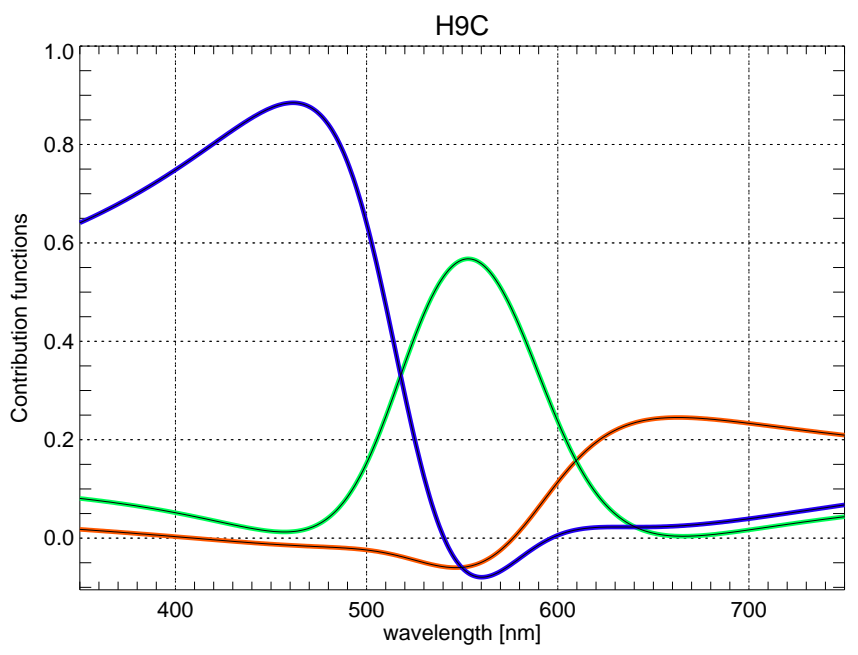

Figure 9. Contribution functions $(D)$ for the ICX285AQ with smoothing parameter $\mu=1$.

for a wide range of visible wavelengths with a fixed smoothing parameter $(\mu=1)$. The contribution functions $(D)$ are presented in Fig. 9, and clearly reflect the three RGB channels of the filter mosaic. For example, estimating the true spectral intensity at $550 \mathrm{~nm}$ is accomplished using primarily the green channel, with minimal contributions from the other two. At wavelengths further away from the filter transmission peaks, retrieval involves a mixture of two or more channels. For example, the best estimate for spectral intensity at $520 \mathrm{~nm}$ is obtained by combining roughly equal amounts of the blue and green channels. This would appear to suggest that useful spectral estimates can be determined for any arbitrary wavelength. 


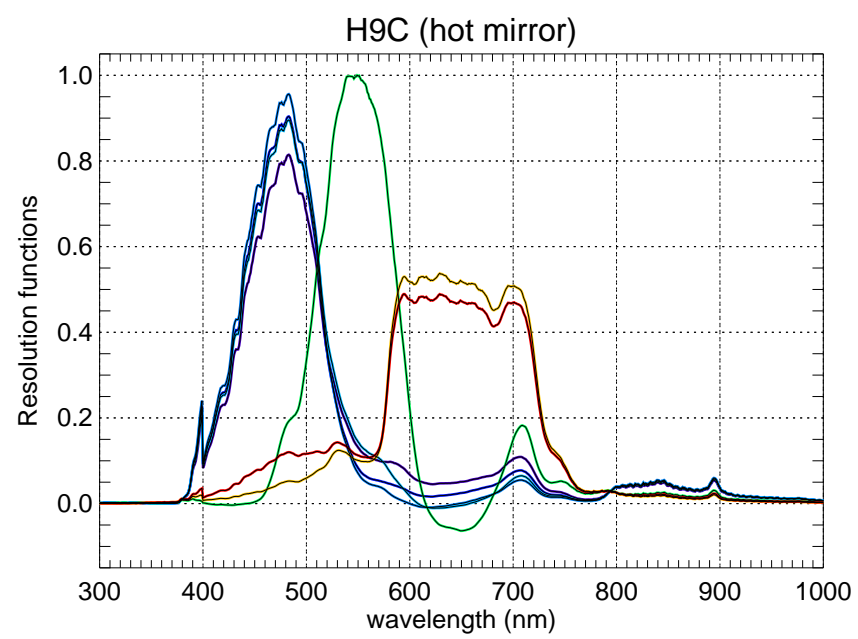

Figure 10. Resolution/window functions $(A)$ for ICX285AQ with smoothing parameter $\mu=1$ and retrieved wavelengths of 391.4 (dark blue), 427.8 (blue), 470.9 (light blue), 486.1 (cyan), 557.7 (green), 630.0 (orange), and $777.7 \mathrm{~nm}$ (red).

Practical limitations become apparent after examining the resolution $(A)$ functions. Figure 10 shows resolution functions calculated for several different auroral emissions from Table 1. There should ideally be seven distinct resolution functions, one centered on every desired wavelength, and each only a few nanometers in width. This would allow us to make independent estimates of the luminosity at each wavelength without any contamination from nearby emissions.

The actual outcome is only three sets of resolution functions with widths of 50-100 nm. All of the blue wavelengths produce nearly identical resolution functions, meaning that it is impossible to distinguish between four of the most commonly considered auroral emissions. More usefully, the green wavelength has a resolution function that is only minimally sensitive to the brightest blue and red emissions. Like the blue emissions, results for different red-line and NIR emissions are effectively indistinguishable.

For wavelengths near the center of each channel the effective resolution is very similar to the corresponding filter transmission curve. However, attempted retrieval at intermediate wavelengths is completely ineffective, producing resolution functions that are essentially the same as one of the primary colors. The underlying measurements simply do not contain enough information to produce different resolution functions away from the three channel centers.

The overall pattern of how resolution functions change with retrieval wavelength can be represented as a twodimensional grid, where each column is the resolution function at a particular wavelength. Figure 11a contains a contour plot of this resolution surface for the H9C camera. This clearly shows that there are three distinct sets of synthetic passband functions, all with full-width half-maximum
(FWHM) on the order of $100 \mathrm{~nm}$, centered near 480, 530 and $620 \mathrm{~nm}$ respectively.

These results indicate that the H9C can only usefully distinguish between three independent spectral ranges, each strongly resembling one of the measurement kernels. More precise wavelength discrimination, such as trying to separate $N_{2}^{+}(470.9 \mathrm{~nm})$ and $H_{\beta}(486.1 \mathrm{~nm})$ emissions, is simply not possible given the measurement characteristics of this device. In the absence of other spectral information, linear inversion will not provide any advantage over working directly with the original RGB instrument channels.

\subsection{ICX429AKL (MX7C)}

Backus-Gilbert analysis applied to the ICX429AKL "fast"mode measurement kernels in Fig. 5 produces resolution functions as shown in Fig. 12. These results are similar to those from the H9C, with only three distinctly resolvable channels. The blue channel is slightly narrower, green is slightly wider, red is very similar, and the resolution contours in Fig. $11 \mathrm{~b}$ are essentially the same.

Analysis of the "raw"-mode kernels from Fig. 3 produces resolution functions as shown in Fig. 13. While there are still only three distinct resolution functions, the blue channel is significantly narrower than for the "fast" mode. Resolution contours in Fig. 11c are also substantially improved. Access to data from all four native color channels on the ICX429AKL is clearly superior to "fast"-mode or threechannel ICX285AQ observations.

Both ICX429AKL modes produce multiple channels with complicated spectral responses. Linear inversion determines how these channels should be combined in order to achieve the best possible wavelength resolution. The transformed channel combinations for this device are easier to work with than the original channels, as they can be used to construct RGB images for visual examination, or directly compared with emission spectra to determine sensitivity.

\subsection{Infrared rejection}

As mentioned in Sect. 3.4, the Rainbow imagers operate with a hot mirror to reject infrared wavelengths. Consequently, most analysis in this study has been carried out assuming the presence of such a filter. For completeness we also studied the effects of including the entire $300-1000 \mathrm{~nm}$ wavelength range covered by our calibration.

These results are shown in Fig. 14 and exhibit significant differences from those obtained using only the visible spectrum. The reason for this is that Backus-Gilbert analysis attempts to minimize window function amplitude by combining different measurement kernels. However, all CCD channels have very similar response curves above $750 \mathrm{~nm}$. The outcome for a $\mathrm{H} 9 \mathrm{C}$ system is clearly suboptimal, with all cases containing significant NIR contributions. MX7C fastmode results are similarly degraded. Interestingly, the MX7C 

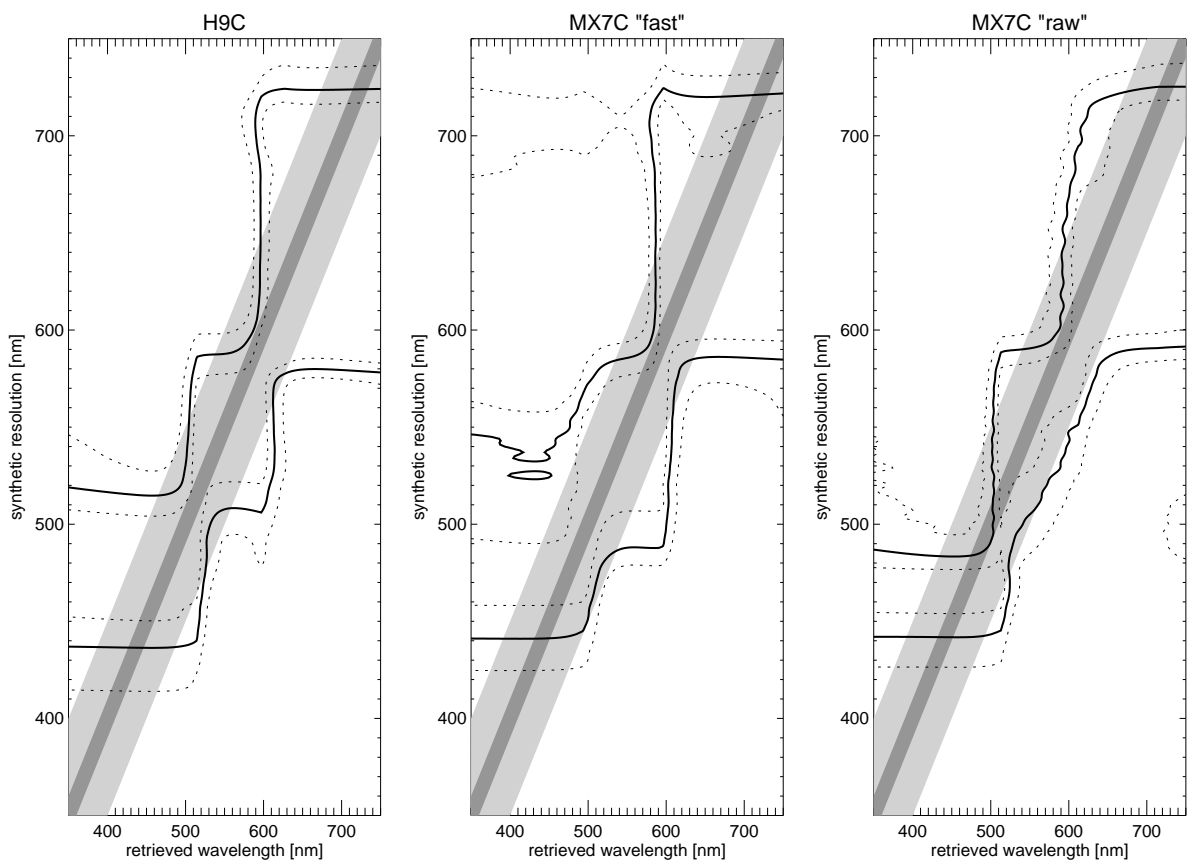

Figure 11. Contour plots of synthetic bandwidth ( $y$ axis) variation with retrieved wavelength ( $x$ axis). All functions are normalized to unit peak; FWHM is indicated by the solid contour. Light and dark diagonal shading corresponds to an unbiased detector with FWHM of 100 and $20 \mathrm{~nm}$ respectively.

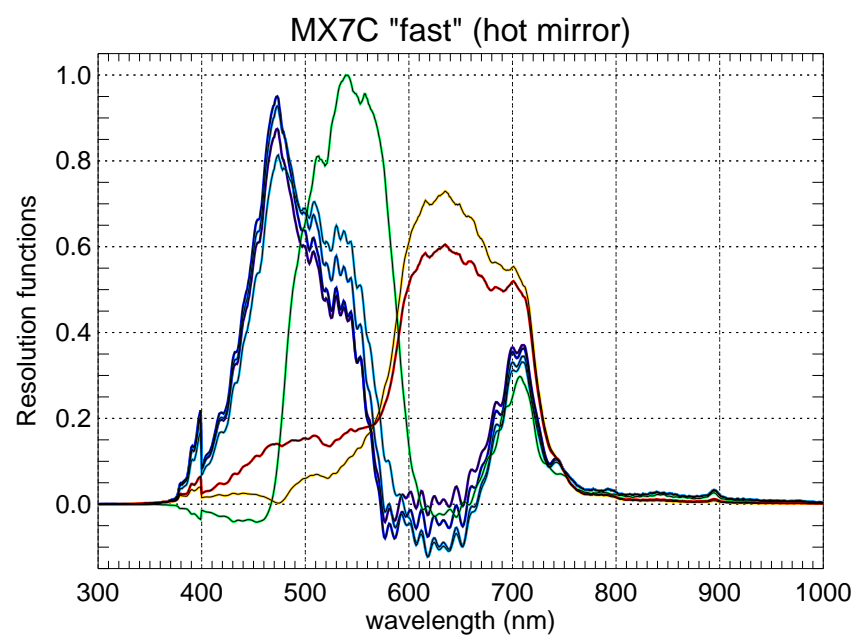

Figure 12. Resolution/window functions $(A)$ for ICX429AKL in "fast" mode with smoothing $\mu=1$ and retrieved wavelengths of 391.4 (dark blue), 427.8 (blue), 470.9 (light blue), 486.1 (cyan), 557.7 (green), 630.0 (orange), and $777.7 \mathrm{~nm}$ (red).

raw mode produces essentially the same blue, green, and red channels as before, but with the addition of a more distinct NIR channel. Backus-Gilbert analysis indicates that NIR filter has important benefits for H9C and MX7C fast-mode data, but eliminates MX7C-raw information.

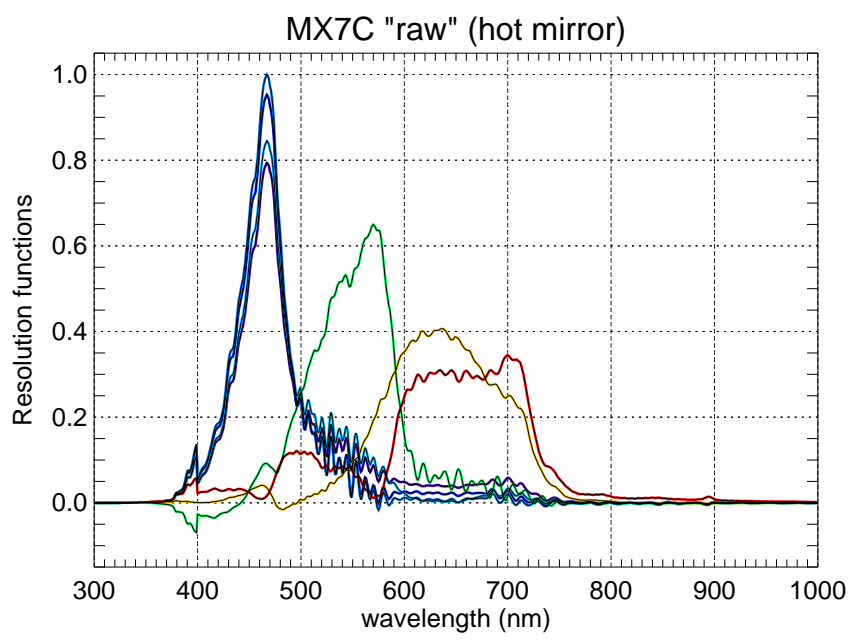

Figure 13. Resolution/window functions $(A)$ for ICX429AKL in "raw" mode with smoothing $\mu=1$ and retrieved wavelengths of 391.4 (dark blue), 427.8 (blue), 470.9 (light blue), 486.1 (cyan), 557.7 (green), 630.0 (orange), and $777.7 \mathrm{~nm}$ (red).

\subsection{RGB estimation}

The central theme of this study is the examination of color mosaic CCD capabilities for spatially resolved multispectral analysis of the aurora. It is admittedly also important to be able to produce colorful images for scientific communication and public outreach. RGB data can often be displayed 

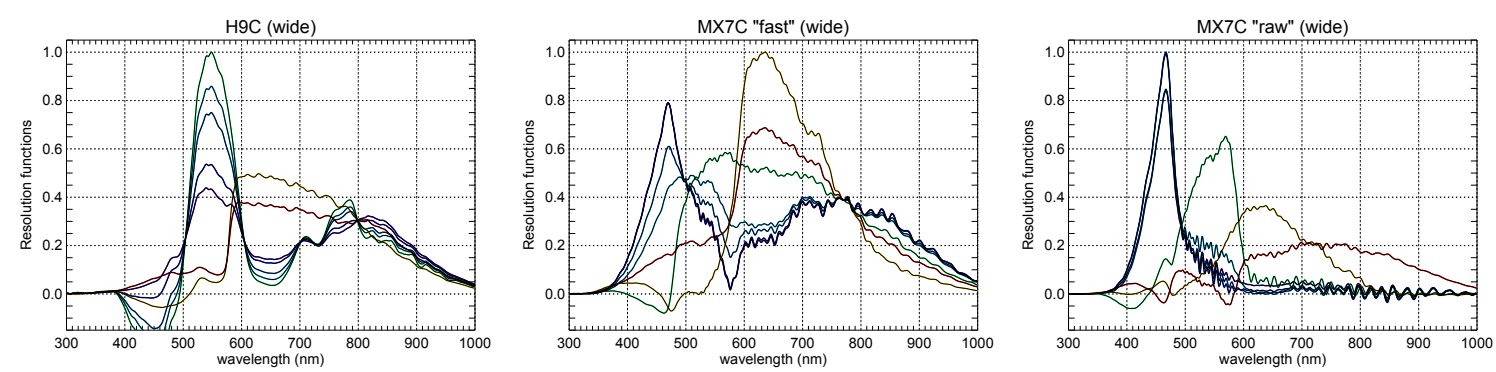

Figure 14. Resolution/window functions $(A)$ without an infrared-blocking hot mirror.

with minimal processing, but CYGM data must first be transformed into some other form. One approach suggested in the ICX429AKL data sheet is reviewed in Appendix B. It relies on the combination of several color theory concepts, and when applied to auroral data the results are often not visually appealing.

As part of this study we explored a very simple alternative using Backus-Gilbert methods to retrieve wavelengths corresponding to each of the three primary colors. In other words, we determine a contribution matrix $\mathbf{D}$ as in Eq. (5) that will produce a color triplet $\mathbf{c}$ from an arbitrary set of measured channel intensities $\mathbf{m}$. The primary colors are selected to match CIE (International Commission on Illumination) 1931 values (Wyszecki and Stiles, 2000).

$\mathbf{c}=\left[\begin{array}{c}700.0 \mathrm{~nm} \\ 546.1 \mathrm{~nm} \\ 435.8 \mathrm{~nm}\end{array}\right]$

For a ICX285AQ CCD the RGB contribution matrix has the relatively simple form given in Eq. (18). The matrix is approximately diagonal, so each primary color is dominated by a single channel, with a scale difference that reflects higher sensitivity to red wavelengths and lower sensitivity to blue.

$\mathbf{c}=\left[\begin{array}{lll}+0.27631 & +0.01982 & +0.04673 \\ -0.07100 & +0.65894 & -0.04815 \\ -0.00980 & +0.02675 & +1.00000\end{array}\right]\left[\begin{array}{l}\mathrm{R} \\ \mathrm{G} \\ \mathrm{B}\end{array}\right]$

The RGB contribution matrix for the MX7C fast mode is given in Eq. (19). There is a similar scale difference reflecting greater CCD sensitivity to red wavelengths, but the matrix is nondiagonal due to the more complex relationship between device channels and the primary colors.

$$
\left[\begin{array}{llll}
-0.03366 & +0.06239 & +0.14099 & -0.09326 \\
+0.13351 & -0.30245 & +0.04012 & +0.37995 \\
-1.00000 & +0.07095 & +0.41522 & +0.81462
\end{array}\right]\left[\begin{array}{c}
\mathrm{Gr}+\mathrm{Ye} \\
\mathrm{Mg}+\mathrm{Cy} \\
\mathrm{Mg}+\mathrm{Ye} \\
\mathrm{Gr}+\mathrm{Cy}
\end{array}\right]
$$

The RGB contribution matrix for the MX7C raw mode is given in Eq. (20). There is a similar scale difference reflecting greater CCD sensitivity to red wavelengths and a complex mapping from device channels to primary colors.

$$
\left[\begin{array}{llll}
-0.14878 & -0.06312 & +0.16537 & +0.15974 \\
+0.30838 & +0.25822 & -0.26835 & -0.25324 \\
+1.00000 & -0.02615 & -0.97716 & +0.02779
\end{array}\right]\left[\begin{array}{c}
\mathrm{Cy} \\
\mathrm{Ye} \\
\mathrm{Gr} \\
\mathrm{Mg}
\end{array}\right]
$$

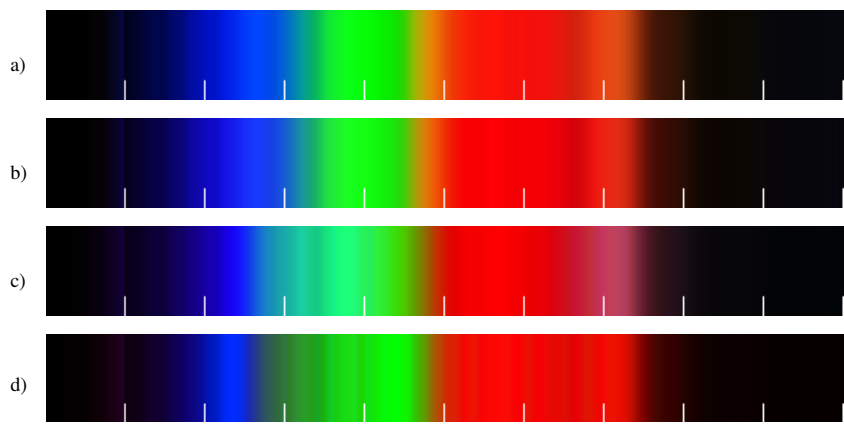

Figure 15. Color bars for calibration wavelengths from 350 to $850 \mathrm{~nm}$ with tick marks at $50 \mathrm{~nm}$ intervals. Top bar (a) is direct mapping of ICX285HQ Bayer matrix to RGB. Lower three are BackusGilbert estimation of CIE RGB channels for (b) ICX285HQ, (c) ICX429AKL fast mode, and (d) ICX429AKL raw mode.

Figure 15 contains a summary of the RGB conversion matrices applied to the calibration scans. These results should be assessed in terms of their visual appearance, so they are displayed as color bars. The top result was produced using direct mapping from Bayer pixels to RGB (i.e., $\mathbf{D}$ is the identity matrix); the other three were calculated using Eqs. (18), (19), and (20). As found previously, linear inversion of ICX285HQ does not provide any improvements relative to the native RGB channels. Both ICX429AKL modes provide similar results, although green extends to shorter wavelengths than for the ICX285HQ. The resulting deficit in blue suggests that additional color channel balancing might help produce more visually appealing images.

\subsubsection{Estimation error}

The estimation error $\epsilon$ due to measurement noise $n$ can be calculated for any specific situation using Eq. (13). Assuming roughly equal noise $n$ in each measurement channel leads to a useful approximation,

$\epsilon_{i}=\sqrt{\Sigma_{j} d_{i j}^{2} n_{j}^{2}} \approx n \sqrt{\Sigma_{j} d_{i j}^{2}} \quad n_{j} \sim n$,

that indicates how sensitive an estimated value is to the presence of measurement noise. As shown in Table 4, the three 
Table 4. Noise sensitivity as indicated by the rms (root mean square) value of coefficients in $\mathbf{D}$ matrix rows.

\begin{tabular}{cccc}
\hline & $\mathrm{R}$ & $\mathrm{G}$ & $\mathrm{B}$ \\
\hline H9C & 0.2809 & 0.6645 & 1.0000 \\
fast & 0.1833 & 0.5052 & 1.3568 \\
raw & 0.2810 & 0.5458 & 1.3978 \\
\hline
\end{tabular}
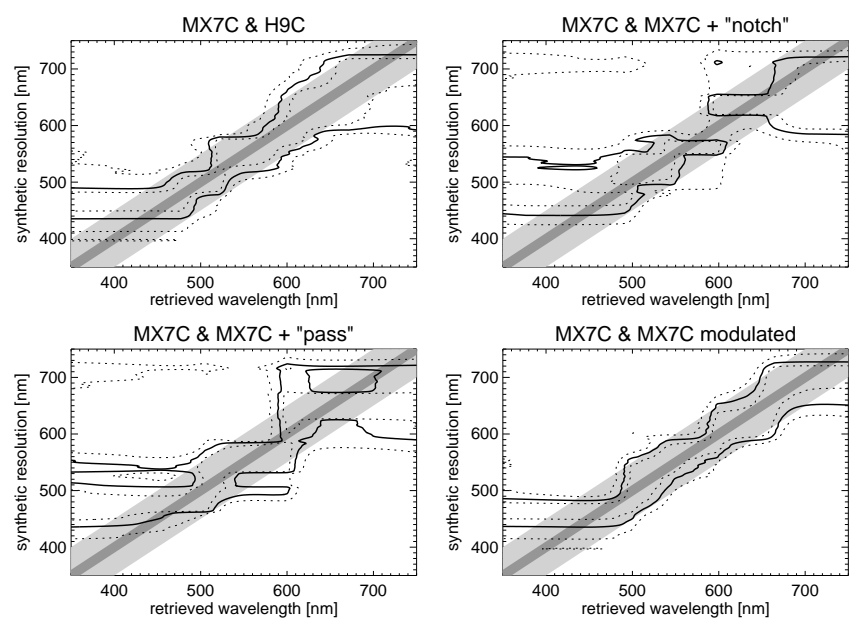

Figure 16. Backus-Gilbert spectral response analysis for combinations of two-color CCDs and filters.

different cases (corresponding to Eqs. 18, 19, 20) are all roughly the same for each color channel.

\section{Combinations and modifications}

Analysis of existing color cameras shows that a four-channel system has better spectral discrimination than a threechannel system. Additional resolution could presumably be obtained by including more spectral channels. For a single $\mathrm{CCD}$ this might be accomplished with a larger number of different microfilters, e.g., a $3 \times 3$ mosaic of nine different color channels. Unfortunately, such devices are not currently available. There are, however, some interesting alternatives based on combinations and modifications of existing technology.

An obvious first step is to combine the two different CCDs considered in this study. While both are effectively threechannel devices, there are enough differences between the filter response profiles to suggest that such a combination would be worthwhile. This turns out to be the case, as can be seen from results of a H9C/MX7C (fast mode) BackusGilbert analysis shown in Fig. 16a. Using seven different filters provides at least four distinct spectral bands, with bestcase resolution on the order of $50 \mathrm{~nm}$.

Further improvements would require more color channels with different spectral response. However, virtually all available commercial CCDs use either RGB or CYGM color mo- saics. One exception is the Sony ICX456 with a RGBE (RGB emerald) mosaic, but the standard output appears to have only 3 RGB channels. Even if direct access to the sensor were possible, it would only provide a single additional channel, and thus only marginally enhance spectral resolution.

There are also very significant challenges associated with merging images from two or more different types of cameras. It may be difficult to synchronize exposure and readout times. Precise calibration is required to correct for different sensitivities. Pixel size and density differences will complicate the process of spectral retrieval at common locations.

It would be easier to combine data from two or more identical cameras, although for effective spectral retrieval it would of course be necessary to have different passbands for each system. One approach would be to simply shift one set of filter responses by some $\Delta \lambda$ chosen to maximize effective resolution. However, this is not an option for wide-band color mosaic filters, unlike narrowband interference filters, which can be tilted to produce minor shifts to longer wavelengths.

The combination of a broadband CCD and a narrowband transmission filter (Fig. 17a) is commonly used in auroral studies to isolate specific emission lines. Additional measurements at nearby wavelengths allow estimation and removal of background light. Equivalently, spectral intensity at each wavelength could be determined by taking the difference between unfiltered broadband signal levels and those obtained using a narrowband rejection (notch) filter (Fig. 17b).

Measuring brightness at several different wavelengths with a single wide-band CCD (Fig. 17c) requires the use of multiple filters or a single tunable filter (López-Alvarez et al., 2008; Sigernes et al., 2012; Goenka et al., 2013). In either case, the measurement process will involve a sequence of observations that are intrinsically nonsimultaneous. This may be a significant problem for sources, such as the aurora, that are often highly dynamic over short timescales. An obvious solution would be to simultaneously operate two or more systems with identical CCDs and different filters. Successful examples of this approach include the all-sky polar camera (PoCa; Steele and Cogger, 1996) using two cameras each with a five-channel filter wheel and the auroral structure and kinetics narrow field imager (ASK; Dahlgren et al., 2008) using three cameras each with a single filter. However, truly simultaneous operation of multiple CCDs produces large amounts of data and has other complications that increase with the number of different wavelengths.

An novel alternative takes advantage of the fact that a single complex filter can have a different effect on each channel of a color camera. For example, consider a single filter with three narrow passbands at 427.8, 557.7, and $630.0 \mathrm{~nm}$. This could be combined with a single RGB CCD to create a " $3 \times 3$ " system (Fig. 17d) that provides three simultaneous narrowband channels.

Compared to a conventional system with a white-light $\mathrm{CCD}$ and a single narrowband filter, the $3 \times 3$ system will 


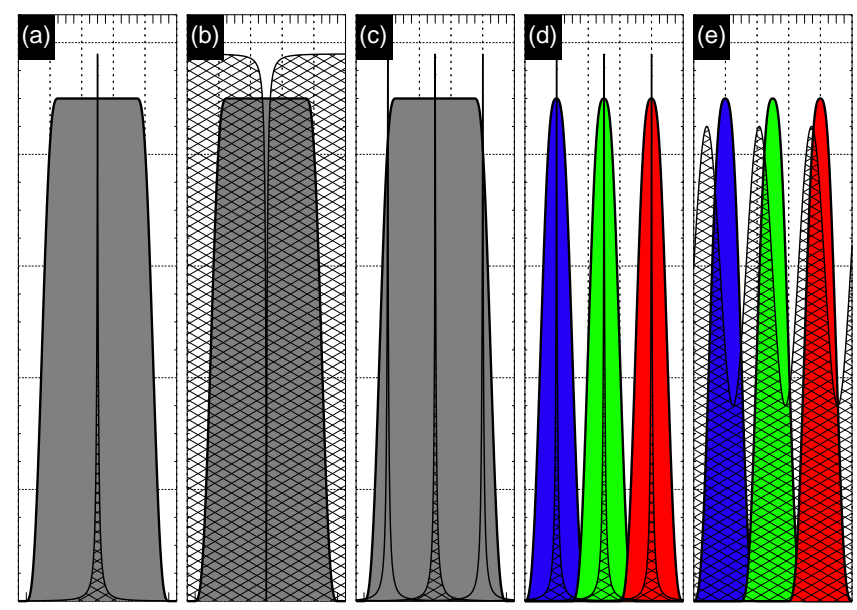

Figure 17. (a) Broadband CCD and narrowband transmission filter, (b) broadband CCD and narrowband rejection (notch) filter, (c) broadband CCD and three different narrow passband transmission filters, (d) three-channel wide-band color CCD and a single transmission filter with three narrow passbands, (d) three-channel wide-band color CCD and a single modulated transmission filter.

have moderately reduced sensitivity $(\sim 80 \%)$ due to the additional wide-band filter losses, significantly reduced sensitivity $(33 \%)$ since only one-third of the pixels respond to each wavelength, and a similar factor-of-three reduction in spatial resolution. Each channel may also contain some leakage from the other two wavelengths, so inversion will be required for optimal signal separation.

For a fair comparison, the conventional system should also measure three different wavelengths, presumably with some kind of filter wheel. This would reduce sensitivity by more than a factor of three, due to additional switching and readout time required for each filter, and data from different filters would not be simultaneous. Overall, the $3 \times 3$ design will have comparable sensitivity and perfect simultaneity, but reduced spatial resolution and inferior out-of-band wavelength rejection.

Phenomena such as quiet auroral arcs, diffuse aurora, polar cap arcs, and patchy pulsating aurora are easily observed by existing Rainbow systems with $5 \mathrm{~s}$ exposures at a $6 \mathrm{~s}$ cadence. Moderate losses from a second filter would not drastically reduce the threshold of observable luminosity. A $3 \times 3$ device should thus be particularly useful during periods with bright dynamic aurora, although the lack of background estimates could be a concern during quiet times.

Independent estimates of signal and background could be obtained with two identical RGB CCDs and a single multinotch filter that rejects three different narrow passbands. One detector would measure signal plus background in three wide-band channels, while the other measures three wide-band background channels with minimal signal contributions. The difference between images from these two detectors will thus contain three channel differences, each of which can be used to infer the background-corrected signal intensity at a different notch wavelength.
Custom multifeature narrowband filters may be relatively expensive compared to the cost of a single color camera. It is reasonable to ask, what can be accomplished using lower cost components? To answer this question we started by considering a stock multinotch filter (Semrock NF01405/488/561/635) with a spectral transmission profile as shown in Fig. 18. A hypothetical two-camera (both MX7C fast mode) system with one multinotch filter was subjected to Backus-Gilbert analysis, with results as shown in Figure 16b. As expected, enhanced spectral resolution $(<40 \mathrm{~nm})$ can be obtained at three notch wavelengths, and moderate improvement $(>50 \mathrm{~nm})$ in several other ranges. For comparison, a similar analysis was carried out using a hypothetical combination of two MX7C cameras and a different filter with multiple passbands (Semrock FF01-440/521/607/694). Results shown in Fig. 16c indicate a similar enhancement in resolution, albeit at a different set of wavelengths.

We explored several other options for dual camera systems. One of the more successful configurations did not require abrupt notches or passbands, but instead used a gradually modulated sinusoidal transmission profile (Fig. 17e) given in Eq. (22).

$$
\frac{1}{4} \sin \left(\frac{\lambda}{\lambda_{0}}+\phi_{0}\right)+\frac{3}{4} \quad \lambda_{0}=17.0 \mathrm{~nm}, \quad \phi_{0}=0
$$

While this particular filter was not selected from a cata$\log$, we do expect that a custom order might not be prohibitively expensive, especially given that the specific details do not drastically alter the outcome. It appears that the spectral response must only be sufficiently different (quasiorthogonal) compared to the other kernels. Results shown in Fig. 16d clearly indicate that this simple combination is even better than a more complicated $\mathrm{H} 9 \mathrm{C} / \mathrm{MX} 7 \mathrm{C}$ pair, providing $<100 \mathrm{~nm}$ resolution over a wide range of visible wavelengths. 


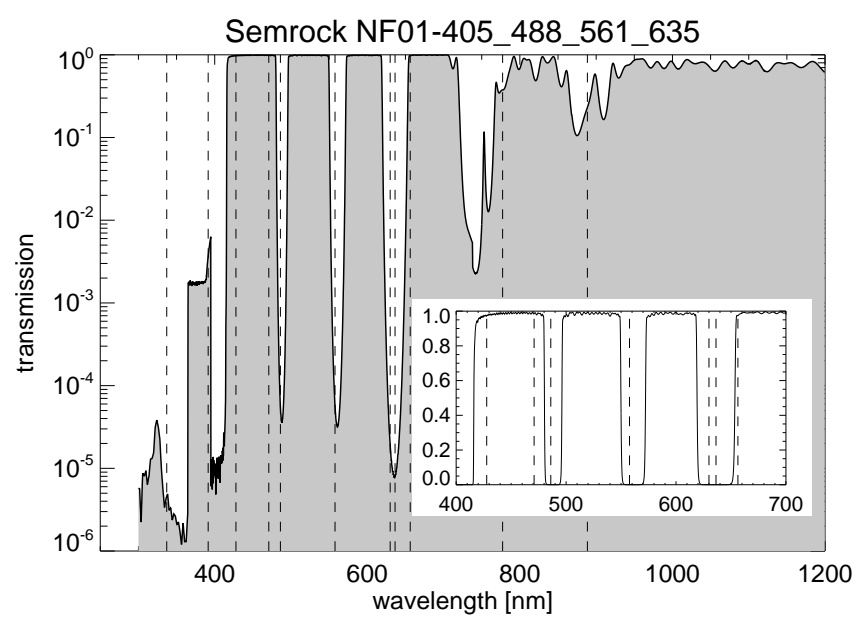

Figure 18. Spectral transmission for a Semrock NF01405/488/561/635 multinotch filter with rejection bands near 405, 488,561 , and $635 \mathrm{~nm}$. Vertical dashed lines correspond to some typical auroral emission wavelengths.

The obvious next step is to consider combinations of three cameras. Two MX7C fast-mode systems using different notch filters and a third unmodified device produce resolution functions as shown in Fig. 19a. This configuration is only slightly better than either notch filter separately, although it is likely that a custom configuration could be significantly better. For comparison we analyzed a three-camera system with simple wavelength shifts of $\Delta \lambda= \pm 9 \mathrm{~nm}$ and obtained results shown in Fig. 19b. This purely hypothetical combination provides $<100 \mathrm{~nm}$ resolution over most of the visible spectrum. Comparable results (Fig. 19c) were also produced by a more physically realistic combination of two sinusoids with different periods and phase offsets $\left(\lambda_{1}=17.0 \mathrm{~nm}, \phi_{1}=-\pi / 4, \lambda_{2}=21.0 \mathrm{~nm}, \phi_{2}=+\pi / 4\right)$.

\subsection{Quantitative comparison}

Contour plots of the window functions, such as those shown in Fig. 19, provide a useful picture of the spectral resolution for a hypothetical system of filters and detectors. However, they are less well suited for quantitative comparison of two or more different systems. For that, we consider certain key Backus-Gilbert parameters as a function of wavelength. Two useful parameters have already been introduced: spectral resolution given by the "spread" ( $q_{2}$ defined in Eq. 14) and sensitivity to measurement error given by the "noise" parameter (Eq. 13). We also introduce a third useful quantity, "bias" $\left(q_{3}\right)$, to measure whether window functions are offset from the retrieval wavelength:

$q_{3}(\lambda)=\int\left(\lambda-\lambda^{\prime}\right) A\left(\lambda, \lambda^{\prime}\right) d \lambda^{\prime}$.

Figure 20 shows how these three parameters vary as a function of wavelength for several of the cases considered
Table 5. Average spread, error, and bias over the wavelength range $450-650 \mathrm{~nm}$ for different cameras and filter combinations.

\begin{tabular}{lccc}
\hline & Spread [nm] & Error & Bias [nm] \\
\hline mx7c-fast & 202.1 & 0.0248 & 29.8 \\
mx7c-raw & 97.6 & 0.1001 & 5.7 \\
h9c & 127.3 & 0.0144 & 19.8 \\
9c, 7cf & 86.5 & 0.0374 & 12.2 \\
7cf, 7cf + NF01 & 181.8 & 0.0350 & 29.3 \\
7cf, 7cf + sine & 127.2 & 0.1505 & 14.3 \\
7cd, 7cf + sin1, 7cf $+\sin 2$ & 76.3 & 0.1387 & 8.7 \\
\hline
\end{tabular}

previously. Spectral resolution is generally poor outside the range of visible wavelengths due to low CCD sensitivity below $400 \mathrm{~nm}$ and hot mirror NIR rejection above $750 \mathrm{~nm}$. A single MX7C operating in fast mode provides a worst-case baseline. Substantial improvements in resolution are predicted for a single MX7C-raw device, with further gains for an unmodified MX7C/H9C combination. Simply adding a stock multinotch filter to a pair of cameras provides very little benefit, but a three-camera system with optimized filters can achieve $50 \mathrm{~nm}$ resolution across a wide spectral range.

The noise error curves show the expected tradeoff against resolution. Spectral estimation from single camera systems with three or four channels is relatively stable, while carefully balanced nine-channel combinations are significantly more unstable in the presence of measurement error. This is likely to be the limiting factor to practical applications of multichannel spectral synthesis, especially for low-light configurations.

Initial values for the modulation parameters in Eq. (22) were determined manually. This process was tedious and did not guarantee an optimal outcome. In order to automate the search through parameter space several different metrics were tested, with the best results being obtained for the average bias $\bar{q}_{3}$,

$\bar{q}_{3}=\frac{\int q_{3}(\lambda) d \lambda}{\int d \lambda}$,

integrated over the wavelength range $450-650 \mathrm{~nm}$. Final values for the single and double modulated configurations were obtained by automated minimization of $\bar{q}_{3}$ starting from several different initial conditions. Table 5 contains a summary of key metrics comparing optimized single- and doublemodulation filters to simpler camera configurations. The results are broadly consistent with previous conclusions, although with some subtle distinctions washed out by spectral averaging.

\section{Discussion}

In this study we examined several aspects of color mosaic CCDs with applications for auroral imaging. The Backus- 

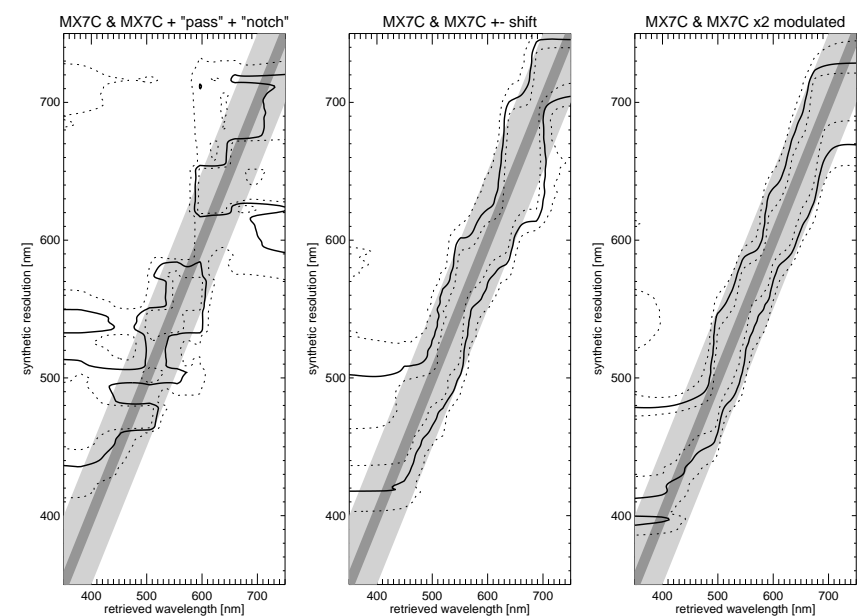

Figure 19. Backus-Gilbert spectral response analysis for combinations of three-color CCDs and filters: (a) two different notch filters, (b) two wavelength shifts of $\Delta \lambda= \pm 9 \mathrm{~nm}$, and (c) two sinusoidal modulations.
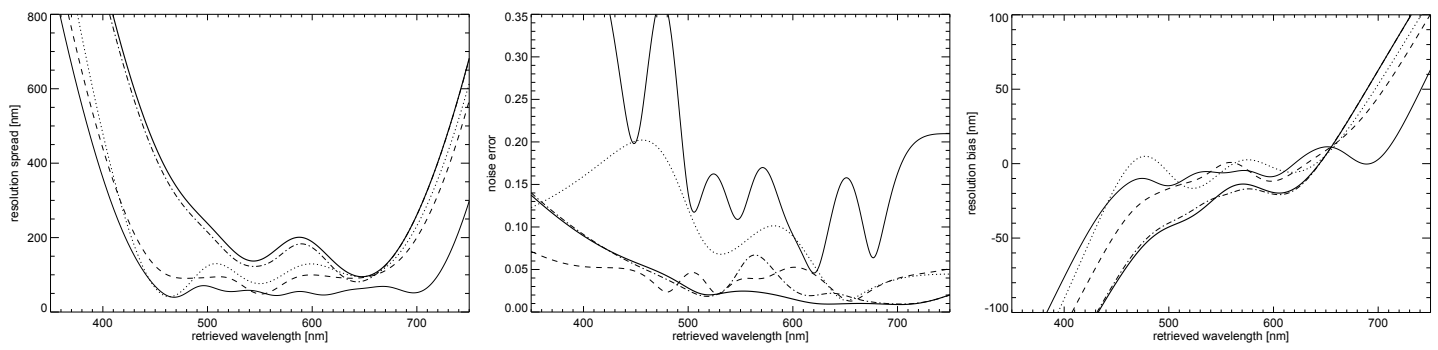

Figure 20. Retrieval resolution $\left(q_{2}\right)$ noise and bias $\left(q_{3}\right)$ for MX7C fast (thick solid line), MX7C raw (dotted), H9C/MX7C (dashed), $2 \times$ MX7C with multinotch filter (dash-dot), and $3 \times$ MX7C with two sinusoidal filters (thin solid).

Gilbert formalism was used as a quantitative basis for instrument comparison and optimization. It also provided a simple method for converting native device channels to RGB.

One motivation for this study was to compare the spectral response of a Sony ICC429AKL CCD for native ("raw") and standard ("fast") readout modes. Results in Fig. 11b and c clearly show that data from both modes can effectively distinguish between only three different wavelength ranges. The raw mode does provide slightly better resolution for blue and green wavelengths, as can also be seen by comparing Figs. 12 and 13. It is also better able to resolve different color bands in the absence of a NIR blocking filter, as shown in Fig. 14. Based only on these considerations, the logical choice would be to acquire data in raw mode whenever possible.

As a practical counter argument, the existing MC7C camera configuration does not appear to support direct readout of the full CCD. A fairly good approximation ("pseudoprogressive", see Appendix A) may be possible, but provides color channels that are not exactly simultaneous. The importance of this effect will depend on the ratio of exposure to readout time, and could be an issue for rapid auroral variations. A pseudo-raw image frame data stream would also be double the size of fast-mode frames, requiring increased operational and financial resources (e.g., storage, telemetry, processing). Still, exploring the feasibility of alternate readout modes appears to be worthwhile.

Results shown in Fig. 11a and b indicate that the H9C spectral resolution is comparable to the MX7C fast mode. Although the ICX285AQ and ICX429AKL CCDs use very different color filter passbands, Backus-Gilbert analysis shows that both devices will produce essentially the same information. This result is perhaps not surprising, as both CCDs were presumably designed to achieve the same goal: distinguishing between three primary colors.

While the effective spectral response of the two CCDs are very similar, they are not identical. This means that combining data from two different cameras could theoretically provide better wavelength resolution than either device separately. Analysis shows that an ideal MX7C/H9C combination (Fig. 16a) would be able to distinguish between at least four different spectral bands. This significant spectral improvement over a single three-channel system would also be accompanied by an increase in total count rate due to the effective doubling of optical and detector area.

In practice, combining data from two different types of cameras is not a simple process. Even starting with identical 
front-end all-sky lenses, different transfer optics would be required to match the image field-of-view to CCDs of different size. Further differences in pixel size, density, and color mosaic pattern must also be accounted for in order to merge results. Finally, the two cameras must either be identically oriented (which can be difficult to ensure over long durations at remote field sites) or images must be carefully corrected for alignment differences. These issues are not insurmountable, but it is not obvious whether the necessary effort is justified by the enhanced spectral resolution.

Merging data is simpler for cameras with identical CCDs and optics, although this will only produce enhanced spectral resolution if the devices have different response functions. This can be achieved with simple color filters as shown by Valero et al. (2007), who used sequential observations through different acrylic filters (e.g., magneta, orange, green, and blue) to improve the spectral resolution of an RGB camera system. Effective observations of rapidly varying processes, such as the aurora, would require simultaneous measurements using several cameras in order to achieve the same results. In this study we have explored the effects of more complex (i.e., multinotch) filters that modify each RGB channel independently. Our results indicate that these can provide better spectral resolution than a single simple color filter, so that fewer cameras would be required to achieve a desired resolution.

We have also shown that the spectral response of a system consisting of three-color CCDs with two modulated filters could be significantly better than a double- or single-camera system. Since this approach is essentially a spectral decomposition using quasi-orthogonal functions, it is tempting to expect that arbitrarily good spectral resolution could be obtained with a sufficiently large number of cameras with different filters. In practice, high-order spectral synthesis would be very sensitive to measurement noise, and critically dependent on precise determination of the different measurement kernels. Multiple wide-band color mosaic camera systems may never be functionally equivalent to a single narrowband device, but they can provide significantly more information than a single color imager, which is in turn more versatile than a white-light device.

Two sets of practical considerations are important for field operation of a multiple camera system. The first set includes issues that scale linearly with number of cameras $N$, i.e., two cameras will cost twice as much, occupy twice the space under a dome, and produce double the volume of data compared to a single camera. The second set consists of issues unique to a spectral synthesis system, such as common orientation, timing, and calibration. It is obviously important that all cameras share the same field of view, but it is relatively easy to achieve this to within $1^{\circ}$ by using a single mounting frame, and more precise alignment can be produced in software using star frames. Millisecond timing accuracy is usually possible with available computers and operating systems, so exposures on the order of $1 \mathrm{~s}$ can be considered to be effectively simultaneous for all but the most dynamic aurora. Calibration is likely to be the most difficult issue, as synthesis depends critically on accurate knowledge of the measurement kernels.

The relative response of different channels on a single CCD can easily be determined with a single wavelength scan, but absolute photometry is essential in order to combine data from different instruments. This requires a more complex calibration procedure than used in this study, but is certainly feasible for experienced personnel with a wellequipped darkroom (e.g., Sigernes et al., 2009). Ongoing monitoring of photometric stability could be carried out using stellar sources, and periodic tests carried out with a field calibration source. This level of effort is rarely justified for a single inexpensive camera, but is not unusual for a highend multichannel narrowband system, and could easily be applied to a spectral synthesis system.

The Backus-Gilbert formalism provides a convenient way to explore the general problem of spectral estimation from a limited set of noisy wide-band measurements. However, it may be useful to consider modifications or alternate (i.e., nonlinear) approaches. This suggestion is motivated by the poor results we obtained when considering the entire spectrum (i.e., no hot mirror). Both the $\mathrm{H} 9 \mathrm{C}$ and MX7C fastmode window functions were highly distorted by the influence of similar NIR sensitivity across all channels. Of course, this behavior is an inevitable response of the analysis algorithm to partially nonorthogonal kernels, and reflects a fundamental lack of information relative to the MX7C raw mode. Still, it might be interesting to explore the effects of altering the definition of spread (Eq. 14) to reduce weighting for out-of-band contributions.

Finally, it may be productive to define the problem more broadly, not just in terms of color channel observations produced by spectral luminosity $s(\lambda)$, but considering the underlying cause: precipitating electrons and ions with a range of energies incident on a realistic atmosphere with parameters $p_{k}$.

$c_{j}=F[s(\lambda)]=G\left[n_{e}(v), n_{i}(v) ; p_{1}, \ldots, p_{k}\right]$

It is of course unreasonable to expect that a few wide-band color channels will be sufficient to invert $G$ and precisely determine the plasma distribution functions $n(v)$. However, a physically appropriate parametrization may result in a tractable problem that could be used to retrieve valuable information about precipitation characteristics near magnetic zenith where perspective effects are minimized. In a followup study we intend to model wide-band response to monoenergetic precipitation and further explore the potential for quantitative inversion of color mosaic imager data.

\section{Conclusions}

Low-cost color mosaic imagers can resolve three relatively wide-band channels that each contain an important auroral 
emission wavelength. A color imager has the capability to provide more information than a single channel white-light system, albeit with reductions in sensitivity and spatial resolution. Although spectral resolution is markedly inferior to cameras using narrowband interference filters, a color mosaic system can provide truly simultaneous acquisition of multiple channels, which is important when observing dynamic aurora. Consumer grade systems can be obtained with shorter lead times, require lower levels of funding, and are typically easier to operate. Multiple low-cost devices can also be installed at numerous locations to span spatial scales that are inaccessible to a single camera.
Combinations of inexpensive color imagers and structured multiband filters can achieve significantly improved spectral resolution. Practical application of this spectral synthesis approach will depend critically on the availability of appropriate filters, and the accuracy of filter and CCD calibration. Further work is also necessary to determine how increased resolution can most usefully be employed for scientific studies.

Color imagers are increasingly widely used in auroral studies, with more than a dozen systems currently in operation. Although their application thus far has been primarily qualitative, they have significant potential for more quantitative spectral analysis. While they cannot compete directly with narrowband systems for auroral spectroscopy, color mosaic cameras could play an important role in advancing our understanding of ionospheric and magnetospheric processes. 


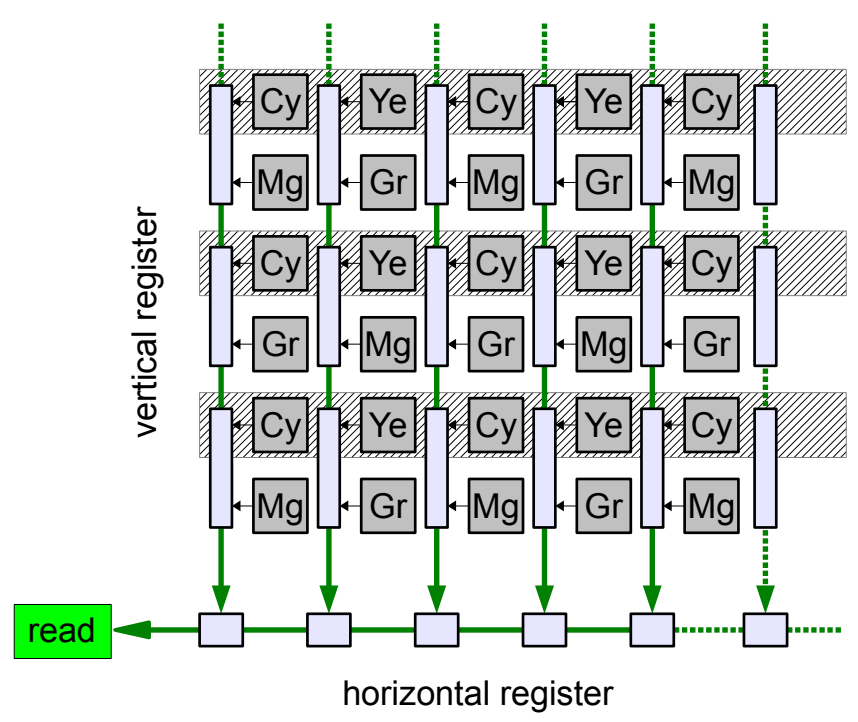

Figure A1. Interline interlaced CMYG CCD geometry.

\section{Appendix A: Interline interlaced CCD}

The Sony ICX429AKL CCD has a standard rectangular array of photosensitive pixels, arranged as in Fig. A1. An additional matrix of wide-band filters provides the ability to produce color images. This CCD is a interline-transfer device with opaque storage registers located between pixel columns. During readout the pixel array contents are shifted very rapidly into the vertical registers, followed by the slower standard sequence of vertical and horizontal clocking. An interline system is sometimes referred to as an electronic shutter, which essentially eliminates image smearing during readout.

The ICX429AKL uses an interlaced readout, meaning that an interline shift can be applied to even or odd rows separately. Either case produces an image frame that has half the vertical resolution of the native ("raw") CCD. Full resolution can be acquired by reading the even and odd frames successively, but the two frames will correspond to different time intervals. An alternative "fast"-mode readout applies an interline shift to both the even and odd rows, but vertical pairs of pixels are combined in a single vertical register, so colors are mixed and vertical resolution is irreversibly halved. In return this mode provides a simultaneous exposure of all image pixels that is not susceptible to time variations in brightness or spectral content.

It is useful to compare the different readout options in quantitative terms. One obvious metric is duty cycle: the maximum fraction of time that can be spent acquiring photons, with higher duty cycle allowing higher sensitivity. For time-varying sources we also care about simultaneity: the amount of overlap between even and odd frames.

The "fast" mode (Fig. A2a) exposes both even and odd rows simultaneously for an interval $T$, combining and read- (a)

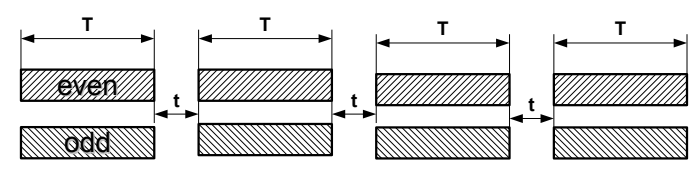

(b)

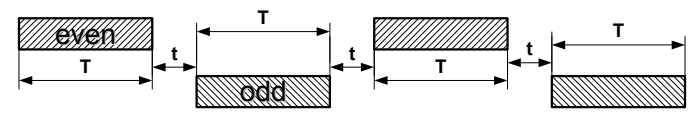

(c)

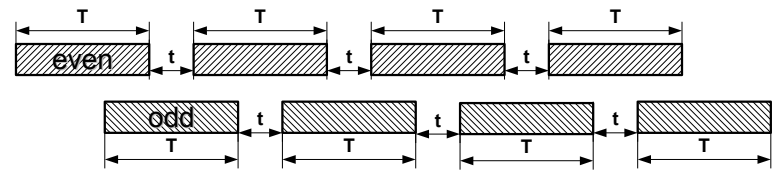

(d)

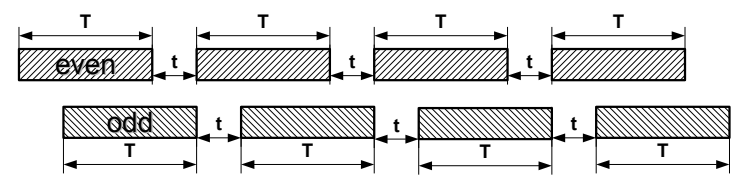

Figure A2. Timing for interlaced readout: (a) "fast mode" with combined simultaneous even and odd rows, (b) alternating nonoverlapping even/odd, (c) alternating overlapping offset, and (d) "pseudo-progressive" alternating minimal offset.

ing them out over a time $t$. The effective duty cycle of $\eta=T /(T+t)$ could approach $100 \%$ for $T \gg t$. Sensitivity results presented in this study were obtained using the nonoverlapping sequence shown in Fig. A2b with a maximum duty cycle $\eta=T /(2 T+t)$ of less than $50 \%$. A lowduty cycle and complete lack of simultaneity are not issues for stable calibration, but can be significant concerns during dynamic auroral observations. The timing sequence in Fig. A2c provides a maximal duty cycle $\eta=T /(T+t)$ but with an overlap $\beta=(T-t) / 2 T$ of less than $50 \%$. The bestcase "pseudo-progressive" exposure sequence in Fig. A2d maximizes duty cycle and overlap $\beta=(T-t) / T$.

The nearly optimal timing sequence in Fig. A2d would produce nearly simultaneous even and odd frames that are shifted by the relatively short readout time $t$. Using the electronic shutter capability it may be possible to (1) start exposing the entire CCD, (2) wait for an interval $t$ then clear and resume exposing the odd frame, (3) finish exposing the even frame and read it out, and (4) finish exposing the odd frame and read it out. This timing sequence would require precise timing control to ensure that even and odd frames were consistently exposed for equal amounts of time.

\section{Appendix B: Sony ICX429AKL YUV/RGB}

The color processing method outlined in the ICX429AKL CCD data sheet (Sony, 2003) uses data from the "fast" readout mode to construct a YUV (luma/chroma) signal. The data sheet does not indicate the specific conversion process from 

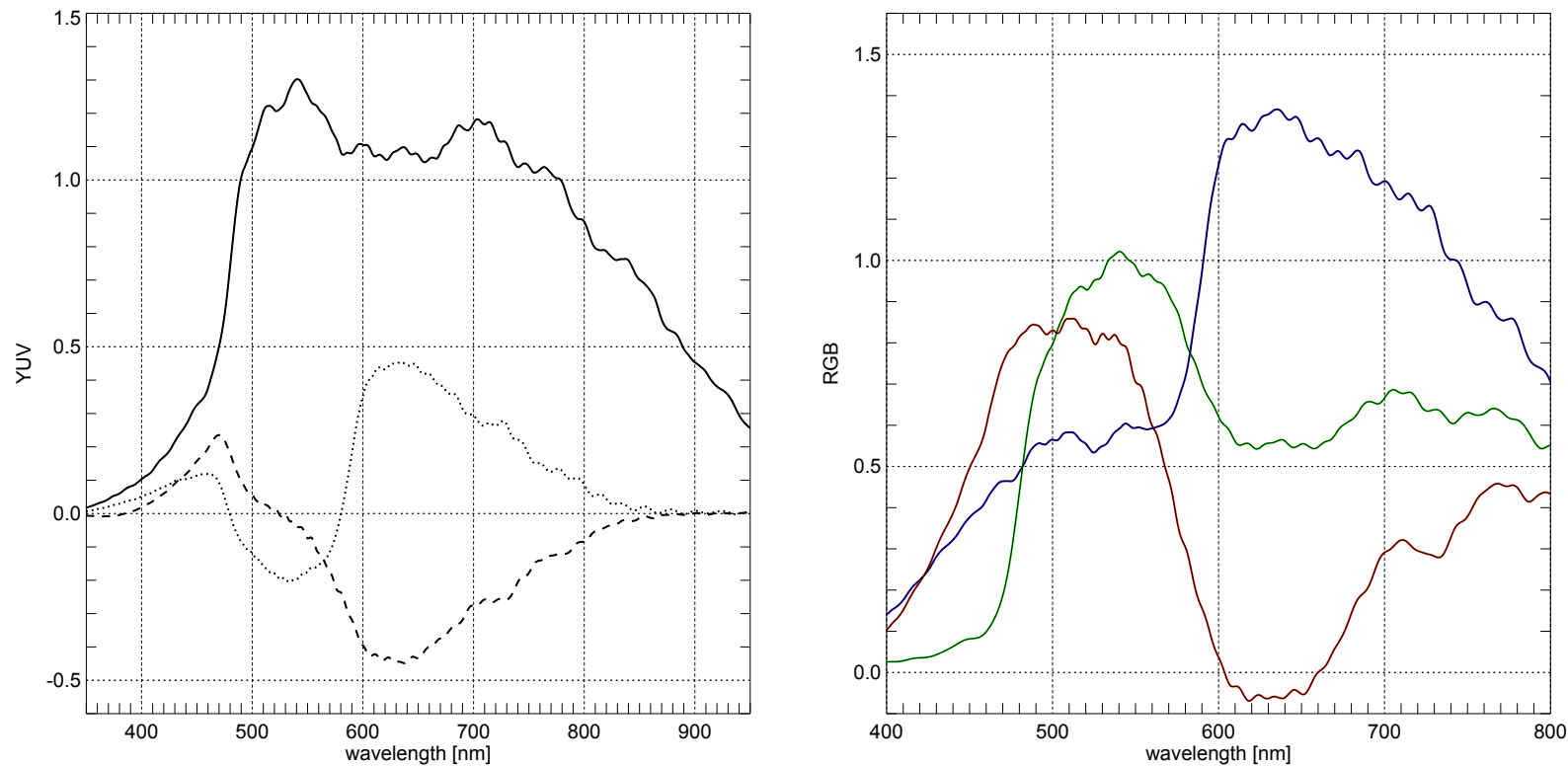

Figure B1. Fast-mode YUV (left) and RGB (right) spectral calibration (no hot filter).

YUV to RGB, so we use definitions from BT601\$2.5 (International Telecommunication Union, 2011):

$$
\begin{array}{ccc}
\mathrm{Y} \equiv & +0.299 \mathrm{R}+0.587 \mathrm{G}+0.114 \mathrm{~B} & {[0.0,1.0]} \\
\mathrm{U} \equiv \mathrm{B}-\mathrm{Y}= & -0.299 \mathrm{R}-0.587 \mathrm{G}+0.886 \mathrm{~B} & {[0.886]} \\
\mathrm{V} \equiv \mathrm{R}-\mathrm{Y}= & +0.701 \mathrm{R}-0.587 \mathrm{G}-0.114 \mathrm{~B} & {[0.701]}
\end{array}
$$

where by convention RGB values are scaled to be in the range $0-1$, producing YUV values between the ranges shown in Eq. (B1). The corresponding inverse transformation from YUV to RGB is given by

$$
\begin{array}{ll}
\mathrm{R}=+1.0 \mathrm{Y}+0.0 \mathrm{U}+1.0 \mathrm{~V} & {[0.0,1.0],} \\
\mathrm{G}=+1.0 \mathrm{Y}-0.194 \mathrm{U}-0.509 \mathrm{~V} & {[0.0,1.0],} \\
\mathrm{B}=+1.0 \mathrm{Y}+1.0 \mathrm{U}+0.0 \mathrm{~V} & {[0.0,1.0] .}
\end{array}
$$

In general it is necessary to consider gamma-corrected quantities $\left(R^{\prime}, G^{\prime}, B^{\prime}, Y^{\prime}\right)$, but for this particular case we simplify matters by using $\gamma=1$.

At this point it is convenient to introduce starred notation (e.g., $Y^{*}$ ) to represent quantities that are in the pixel count range $\left[0, N_{\max }\right]$. Summing horizontally adjacent pixels from ICX429AKL fast-mode data will provide estimates of the brightness (luminance) $Y^{*}$,

$$
Y^{*}=(\mathrm{Gr}+\mathrm{Cy})^{*}+(\mathrm{Mg}+\mathrm{Ye})^{*} \sim 2 B^{*}+3 G^{*}+2 R^{*},
$$

while subtracting horizontally adjacent pixels will provide estimates of the color difference (chrominance) channels $U^{*}$ and $V^{*}$,

$\begin{aligned} U^{*} & =(\mathrm{Mg}+\mathrm{Cy})^{*}-(\mathrm{Gr}+\mathrm{Ye})^{*} \sim 2 B^{*}-G^{*}, \\ V^{*} & =(\mathrm{Mg}+\mathrm{Ye})^{*}-(\mathrm{Gr}+\mathrm{Cy})^{*} \sim 2 R^{*}-G^{*},\end{aligned}$ with different equations applied to the even and odd rows. A $2 \times 2$ pixel block of fast-mode data will be sufficient to determine both $U^{*}$ and $V^{*}$, along with two independent luminance estimates $Y_{1}^{*}$ and $Y_{2}^{*}$.

$$
\left[\begin{array}{l}
Y_{1} \\
Y_{2} \\
U \\
V
\end{array}\right]^{*}=\left[\begin{array}{cccc}
\frac{1}{2} & \frac{1}{2} & 0 & 0 \\
0 & 0 & \frac{1}{2} & \frac{1}{2} \\
+1 & -1 & 0 & 0 \\
0 & 0 & +1 & -1
\end{array}\right]\left[\begin{array}{c}
\mathrm{Gr}+\mathrm{Ye} \\
\mathrm{Mg}+\mathrm{Cy} \\
\mathrm{Mg}+\mathrm{Ye} \\
\mathrm{Gr}+\mathrm{Cy}
\end{array}\right]^{*}
$$

Demosaicing considerations are neglected in this study, so we assume equality of the luminance estimates:

$Y^{*}=\frac{1}{2}\left(Y_{1}^{*}+Y_{2}^{*}\right) \approx Y_{1}^{*} \approx Y_{2}^{*}$,

which can be conveniently expressed in matrix notation.

$$
\left[\begin{array}{c}
\mathrm{Y} \\
\mathrm{U} \\
\mathrm{V}
\end{array}\right]^{*}=\left[\begin{array}{cccc}
\frac{1}{2} & \frac{1}{2} & 0 & 0 \\
0 & 0 & 1 & 0 \\
0 & 0 & 0 & 1
\end{array}\right]\left[\begin{array}{c}
Y_{1} \\
Y_{2} \\
U \\
V
\end{array}\right]^{*}
$$

Using fast-mode response curves from Fig. 5 produces YUV spectral calibration results shown in Fig. B1a. These can be used to rescale pixel count-based $Y^{*} U^{*} V^{*}$ values onto the normalized ranges for YUV given in Eq. (B1),

$$
\left[\begin{array}{l}
\mathrm{Y} \\
\mathrm{U} \\
\mathrm{V}
\end{array}\right]=\left[\begin{array}{ccc}
s_{1} & 0 & 0 \\
0 & s_{2} & 0 \\
0 & 0 & s_{3}
\end{array}\right]=\left[\begin{array}{c}
Y^{*} \\
U^{*} \\
V^{*}
\end{array}\right],
$$

with empirically determined scaling factors of Fig. B1,

$$
\left(s_{1}, s_{2}, s_{3}\right) \approx \frac{1}{N_{\max }}\left(\frac{1}{1.3}, \frac{0.886}{0.45}, \frac{0.701}{0.45}\right),
$$


to get a direct mapping from fast-mode pixel counts to $R^{*} G^{*} B^{*}$ (scaled from 0 to $N_{\max }$ ) as shown in Fig. B1b.

$$
\begin{aligned}
& {\left[\begin{array}{l}
\mathrm{R} \\
\mathrm{G} \\
\mathrm{B}
\end{array}\right]^{*}=\left[\begin{array}{llll}
+0.1923 & +0.1923 & +1.7501 & -1.3655 \\
-0.1897 & +0.5743 & -0.6006 & +0.9852 \\
+2.1612 & -1.7766 & +0.1923 & +0.1923
\end{array}\right]} \\
& {\left[\begin{array}{c}
\mathrm{Gr}+\mathrm{Ye} \\
\mathrm{Mg}+\mathrm{Cy} \\
\mathrm{Mg}+\mathrm{Ye} \\
\mathrm{Gr}+\mathrm{Cy}
\end{array}\right]^{*}}
\end{aligned}
$$


Acknowledgements. Operational support for the Canadian Rainbow network was provided by the Canadian Space Agency. Linux kernel camera driver software was written by David Schmenk. AURIC auroral spectra were provided by Scott Evans at Computational Physics Incorporated. We thank both referees for their very helpful comments.

Edited by: A.-M. Harri

\section{References}

Aster, R. C., Borchers, B., and Thurber, C. H.: Parameter estimation and inverse problems, Elsevier, Burlington, MA, USA, 2005.

Backus, G. and Gilbert, F.: The Resolving Power of Gross Earth Data, Geophys. J. Roy. Astronom. Soc., 16, 169-205, doi:10.1111/j.1365-246X.1968.tb00216.x, 1968.

Backus, G. and Gilbert, F.: Uniqueness In The Inversion Of Inaccurate Gross Earth Data, Philos. Trans. Roy. Soc. London A, 266, 123-192, 1970.

Dahlgren, H., Ivchenko, N., Sullivan, J., Lanchester, B. S., Marklund, G., and Whiter, D.: Morphology and dynamics of aurora at fine scale: first results from the ASK instrument, Ann. Geophys., 26, 1041-1048, doi:10.5194/angeo-26-1041-2008, 2008.

Donovan, E. F., Mende, S., Jackel, B. J., Frey, H., Syrjäsuo, M., Voronkov, I. O., Trondsen, T., Peticolas, L., Angelopoulos, V., Harris, S., Greffen, M., and Connors, M.: The THEMIS allsky imaging array - system design and initial results from the prototype imager, J. Atmos. Solar-Terrest. Phy., 68, 1472-1487, doi:10.1016/j.jastp.2005.03.027, 2006.

Goenka, C., Semeter, J., Noto, J., Dahlgren, H., Marshall, R., Baumgardner, J., Riccobono, J., and Migliozzi, M.: Tunable Filters for Multispectral Imaging of Aeronomical Features, Adv. Space Res., 52, 1366-1377, doi:10.1016/j.asr.2013.06.014, 2013.

Gunturk, B., Glotzbach, J., Altunbasak, Y., Schafer, R., and Mersereau, R.: Demosaicking: color filter array interpolation, IEEE Signal Proc. Magazine, 22, 44-54, doi:10.1109/MSP.2005.1407714, 2005.

Harris, S. E., Mende, S. B., Angelopoulos, V., Rachelson, W., Donovan, E. F., Jackel, B. J., Greffen, M., Russell, C. T., Pierce, D. R., Dearborn, D. J., Rowe, K. M., and Connors, M.: THEMIS Ground Based Observatory System Design, Space Sci. Rev., 141, 213-233, doi:10.1007/s11214-007-9294-z, 2008.

International Telecommunication Union: Studio encoding parameters of digital television for standard 4:3 and wide-screen 16:9 aspect ratios (ITU-R BT.601-7), Tech. rep., International Telecommunication Union, Geneva, 2011.

López-Alvarez, M. A., Hernández-Andrés, J., and Romero, J.: Developing an optimum computer-designed multispectral system comprising a monochrome CCD camera and a liquid-crystal tunable filter., Appl. Opt., 47, 4381-4390, 2008.

Menke, W.: Geophysical data analysis: discrete inverse theory, Academic Press, London, UK, Revised Edn., 1989.

Nieves, J. L., Valero, E. M., Nascimento, S. M. C., HernándezAndrés, J., and Romero, J.: Multispectral synthesis of daylight using a commercial digital CCD camera., Appl. Opt., 44, 56965703, 2005.
Nieves, J. L., Valero, E. M., Hernández-Andrés, J., and Romero, J.: Recovering fluorescent spectra with an RGB digital camera and color filters using different matrix factorizations., Appl. Opt., 46, 4144-4154, 2007.

Parker, R. L.: Inverse Theory with Grossly Inadequate Data, Geophys. J. Int., 29, 123-138, doi:10.1111/j.1365246X.1972.tb02203.x, 1972.

Partamies, N., Syrjäsuo, M., and Donovan, E. F.: Using colour in auroral imaging, Canadian J. Phys., 85, 101-109, doi:10.1139/p06090, 2007.

Partamies, N., Syrjäsuo, M., Donovan, E. F., and Knudsen, D. J.: Dense Array Imaging SYstem prototype observations of missing auroral scale sizes, Proceedings of the 33rd Annual Meeting on Atmospheric Studies by Optical Methods, IRF Sci. Rep, 292, 95 101, 2008.

Partamies, N., Sangalli, L., Donovan, E., Connors, M., and Charrois, D.: Tomography-like Approach for Analysing Colour Auroral Images, Geophysica, 48, 81-90, 2012.

Pulkkinen, T. I., Janhunen, P., Viljanen, A., Pellinen, R. J., Kauristie, K., Opgenoorth, H. J., Wallman, S., Eglitis, P., Amm, O., Nielsen, E., and Thomas, C.: OBSERVATIONS OF SUBSTORM ELECTRODYNAMICS USING THE MIRACLE NETWORK, in: Proceedings of the International Conference on Substorms-4, Vol. 238, 111-114, 1998.

Rees, M. H. and Luckey, D.: Auroral Electron Energy Derived From Ratio of Spectroscopic Emissions, 1. Model Computations, J. Geophys. Res., 79, 5181-5186, doi:10.1029/JA079i034p05181, 1974.

Rodgers, C. D.: Retrieval of atmospheric temperature and composition from remote measurements of thermal radiation, Rev. Geophys., 14, 609-624, 1976.

Rodgers, C. D.: Characterization and Error Analysis of Profiles Retrieved From Remote Sounding Measurements, J. Geophys. Res. 95, 5587-5595, doi:10.1029/JD095iD05p05587, 1990.

Sigernes, F., Holmes, J. M., Dyrland, M., Lorentzen, D. A., Svenø e, T., Heia, K., Aso, T., Chernouss, S., and Deehr, C. S.: Sensitivity calibration of digital colour cameras for auroral imaging, Opt. Express, 16, 15623-15632, 2008.

Sigernes, F., Dyrland, M., Peters, N., Lorentzen, D. A., Sven $\varnothing$ e, T., Heia, K., Chernouss, S., Deehr, C. S., and Kosch, M.: The absolute sensitivity of digital colour cameras, Opt. Express, 17, 20211-20220, 2009.

Sigernes, F., Ivanov, Y., Chernouss, S., Trondsen, T., Roldugin, A., Fedorenko, Y., Kozelov, B., Kirillov, A., Kornilov, I., Safargaleev, V., Holmen, S., Dyrland, M., Lorentzen, D., and Baddeley, L.: Hyperspectral all-sky imaging of auroras., Opt. Express, 20, 27650-27660, 2012.

Sony: ICX429AKL color CCD, 2003.

Steele, D. P. and Cogger, L. L.: Polar patches and the "tongue of ionization”, Radio Sci., 31, 667-677, doi:10.1029/96RS00369, 1996.

Strickland, D. J., Meier, R. R., Hecht, J. H., and Christensen, A. B.: Deducing Composition and Incident Electron Spectra From Ground-Based Auroral Optical Measurements: Theory and Model Results, J. Geophys. Res., 94, 13527-13539, doi:10.1029/JA094iA10p13527, 1989. 
Strickland, D., Bishop, J., Evans, J., Majeed, T., Shen, P. M., Cox, R. J., Link, R., and Huffman, R. E.: Atmospheric Ultraviolet Radiance Integrated Code (AURIC): Theory, software architecture, inputs, and selected results, J. Quant. Spectrosc. Ra., 62, 689$742,1999$.

Tarantola, A.: Inverse problem theory and methods for model parameter estimation, Vol. 130, Society for Industrial and Applied Mathematics, Philadelphia, available at: http://www.ipgp.fr/ $\sim$ tarantola/Files/Professional/SIAM/InverseProblemTheory.pdf, 2005.
Toyomasu, S., Futaana, Y., Yamauchi, M., and Turiststation, A.: Low cost webcast system of real-time all-sky auroral images and MPEG archiving in Kiruna, Proceedings of 33rd Annual European Meeting on Atmospheric Studies by Optical Methods, Kiruna, 292, 75-84, available at: http://www.irf.se/publications/ proc33AM_files/toyomasu-etal.pdf, 2008.

Valero, E. M., Nieves, J. L., Nascimento, S. M. C., Amano, K., and Foster, D. H.: Recovering spectral data from natural scenes with an RGB digital camera and colored filters, Color Res. Appl., 32, 352-360, doi:10.1002/col.20339, 2007.

Wyszecki, G. and Stiles, W. S.: Color science: concepts and methods, quantitative data and formulae, Wiley-Interscience, New York, 2nd Edn., 2000. 